\title{
Typische Problemfelder in Betriebsprüfungen
}

\author{
Thomas Bittner, Roman Dawid und Susann Metzner
}

\subsection{Bedeutung von Verrechnungspreisthemen in Betriebsprüfungen in Deutschland}

Dass Verrechnungspreise sich seit Langem zu einem Dauerthema in Betriebsprüfungen entwickelt haben, ist unbestritten. Bereits 1997 haben Kuckhoff und Schreiber das noch immer für den Praktiker lesenswerte Buch „Verrechnungspreise in der Betriebsprüfung“ veröffentlicht. Sie konzentrieren sich dabei im Wesentlichen auf Warenlieferungen zwischen verbundenen Gesellschaften, weil diese im Mittelpunkt der damaligen Betriebsprüfungspraxis standen ${ }^{1}$.

\footnotetext{
${ }^{1}$ Kuckhoff/Schreiber 1997, VII.
}

Abschnitt 6.1 und 6.2 wurde von Thomas Bittner, Abschn. 6.3 und 6.4 von Susann Metzner und Abschn. 6.5 von Roman Dawid erstellt.

T. Bittner $(\bowtie)$

PricewaterhouseCoopers AG Wirtschaftsprüfungsgesellschaft, Alsterufer 1, 20354 Hamburg, Deutschland

E-Mail: thomas.bittner@de.pwc.com

R. Dawid

PricewaterhouseCoopers AG Wirtschaftsprüfungsgesellschaft, Friedrich-Ebert-Anlage 35-37, 60327 Frankfurt am Main, Deutschland

E-Mail: roman.dawid@de.pwc.com

S. Metzner

Flick Gocke Schaumburg, Rechtsanwälte Wirtschaftsprüfer Steuerberater, Partnerschaft mbB, Brienner Straße 29, 80333 München, Deutschland

E-Mail: susann.metzner@fgs.de

(C) Der/die Herausgeber bzw. der/die Autor(en) 2016

R. Dawid (Hrsg.), Verrechnungspreise, DOI 10.1007/978-3-658-09377-8_6 
Verrechnungspreise stehen auch zwei Jahrzehnte nach der Veröffentlichung von Kuckhoff/Schreiber im Mittelpunkt der Betriebsprüfung, allerdings hat sich das Themenspektrum deutlich ausgeweitet und das Klima in Betriebsprüfungen verschärft. Beide Aspekte werden im Folgenden ausführlicher dargestellt.

Die zunehmende Bedeutung von Verrechnungspreisen in Betriebsprüfungen ist nicht auf Deutschland beschränkt. Zum einen haben immer mehr Länder in den letzten 20 Jahren detaillierte nationale Verrechnungsregelungen erlassen oder bestehende Vorschriften verschärft. Darüber hinaus wird aller Voraussicht nach die Umsetzung der im Zuge der BEPS Initiative überarbeiteten OECD-Richtlinien in nationales Recht zu einem stärkeren Fokus von Betriebsprüfungen auf Verrechnungspreise führen. Insofern dürften die unten stehenden Ausführungen zu aktuellen deutschen Betriebsprüfungen zumindest teilweise repräsentativ für andere Länder sein.

\subsubsection{Themen in deutschen Betriebsprüfungen}

Laut Bundesministerium der Finanzen haben Betriebsprüfungen im Jahr 2014 zu Mehrsteuern und Zinsen in Höhe von 17,9 Mrd. € geführt. ${ }^{2}$ Die steuerliche Mehrbelastung dürfte zu einem nicht unerheblichen Teil aus Verrechnungspreiskorrekturen resultieren, die im Rahmen von Betriebsprüfungen erfolgt sind.

Wie eine Umfrage der PricewaterhouseCoopers AG aus dem Jahr 2015 zeigt, waren Verrechnungspreisthemen (sofern ein Thema des internationalen Steuerrechts Gegenstand der Betriebsprüfung war) der bei weitem häufigste Grund für Einkommenskorrekturen. ${ }^{3}$ Knapp zwei Drittel der Befragten nannten Verrechnungspreiskorrekturen als Grund für steuerliche Mehrbelastungen, während das zweitwichtigste Thema (Quellensteuern auf Lizenzen und Dividenden) von jedem Zehnten als Grund für Einkommenskorrekturen genannt wurde. ${ }^{4}$

Wie Abb. 6.1 zeigt, nannten 63\% der Unternehmen Kostenumlagen und jeweils 57\% Gewinnmargen verbundener Unternehmen bzw. die Dokumentation von Leistungsbeziehungen zwischen verbundenen Unternehmen als Korrekturgrund. Mit deutlichem Abstand aber immer noch auf hohem Niveau folgten die Angemessenheit von Lizenzvergütungen (35\%), die Fremdüblichkeit von Zinsen bei konzerninternen Darlehen oder Cash Pools (26\%) und Funktionsverlagerungen $(21 \%)$.

Die häufige Nennung von Gewinnmargen als Thema in einer Betriebsprüfung verwundert angesichts der in der Praxis überragenden Bedeutung gewinnorientierter Methoden zur Verprobung der Fremdüblichkeit von Verrechnungspreisen nicht.

Hohe Gewinnmargen ausländischer Vertriebs- und Produktionsgesellschaften bzw. Unternehmen mit Dauerverlusten in Deutschland dürften daher zu den häufigsten

\footnotetext{
${ }^{2}$ Vgl. BMF (2015), Ergebnisse der steuerlichen Betriebsprüfung 2014.

${ }^{3}$ Vgl. PwC, Betriebsprüfung 2015; siehe www.pwc.de/BP_2015.

${ }^{4}$ Vgl. PwC, Betriebsprüfung 2015, S. 39.
} 


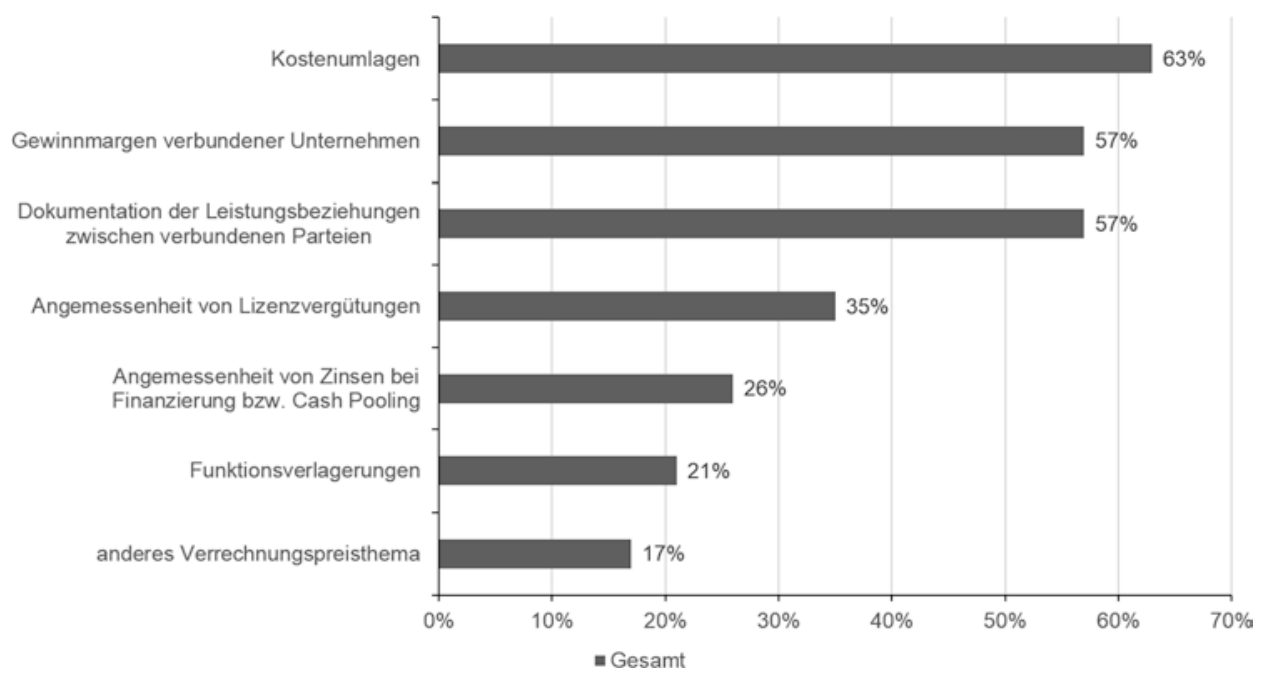

Abb. 6.1 Top Verrechnungspreisthemen in Betriebsprüfungen entsprechend $\mathrm{PwC}$ Betriebsprüfungsstudie 2015

Streitpunkten innerhalb der Betriebsprüfung zählen. Für die Praxis bedeutet dies, dass relativ hohe Margen bzw. Verluste ein zuverlässiger Frühindikator für Diskussionen im Rahmen einer Betriebsprüfung sind.

Demgegenüber erstaunt auf den ersten Blick, dass 57\% der Umfrageteilnehmer als Grund für Mehrbelastungen eine unvollständige Dokumentation der Leistungsbeziehungen zwischen den verbundenen Unternehmen nannten. Immerhin gelten in Deutschland gemäß $\S 90$ Abs. 3 AO Dokumentationspflichten für alle Wirtschaftsjahre, die nach dem 31.12.2002 beginnen. Für den Großteil der Betriebsprüfungsjahre sollte daher grundsätzlich eine Verrechnungspreisdokumentation erstellt worden sein. Möglicherweise sind diese Dokumentationen nicht erstellt worden oder haben nicht die Anforderungen der Betriebsprüfung erfüllt oder die Steuerpflichtigen sind ihren allgemeinen Mitwirkungspflichten nicht nachgekommen. Insgesamt belegt das Umfrageergebnis eindrucksvoll, dass formelle Dokumentationsmängel in der Praxis relevant sind und dass die Betriebsprüfung bei Nichterfüllung der Dokumentationsverpflichtungen durch den Steuerpflichtigen von ihrer erweiterten Schätzbefugnis nach $\S 162$ Abs. 3 AO Gebrauch macht.

Wenig überraschend ist die ausgeprägte Bedeutung von zentralen Kostenumlagen im Rahmen von Betriebsprüfungen und für Verrechnungspreiskorrekturen. Wie in Abschn. 6.4.2 noch ausführlicher dargestellt wird, führen in Betriebsprüfungen neben formellen Fragen (z. B. Anforderungen an vertragliche Grundlagen) auch schwer zu quantifizierende Aspekte wie der Umfang nicht verrechenbarer Gesellschafterkosten oder der Nutzen einzelner Dienstleistungen zu Diskussionen mit der Betriebsprüfung. Darüber hinaus werden in den meisten international tätigen Konzernen zentrale Dienstleistungen erbracht und daher ist dies in fast allen Betriebsprüfungen ein heikles Thema.

Im Gegensatz dazu ist die Vergabe von Lizenzen und Darlehen innerhalb von Konzernen deutlich weniger weit verbreitet. Dennoch sind beide Transaktionen gemäß der 
Unternehmensumfrage häufig Ursache für Verrechnungspreiskorrekturen. Dies spricht dafür, dass auch Lizenzen und Darlehen mittlerweile verstärkt in den Fokus der Betriebsprüfung gerückt sind. Eine Konzentration der Betriebsprüfung auf Warenlieferungen, wie sie Kuckhoff und Schreiber 1997 konstatiert haben, lässt sich heute dagegen nicht mehr feststellen.

Die relativ wenigen Nennungen von Funktionsverlagerungen als Prüfungsthema dürfen nicht zu dem grundsätzlichen Schluss verleiten, Funktionsverlagerungen wären kaum Gegenstand von Betriebsprüfungen. Funktionsverlagerungen finden im Gegensatz zur Verrechnung von Kostenumlagen oder der Verrechnung von Lizenzen in der betriebswirtschaftlichen Praxis weniger häufig statt. Wenn Funktionsverlagerungen durchgeführt werden, sind sie in der Regel auch Gegenstand der Betriebsprüfung.

\subsubsection{Klima in deutschen Betriebsprüfungen}

Trotz der Erhebung von knapp 18 Mrd. € Mehrsteuern im Jahr 2014 berichtet ein relativ geringer Anteil der befragen Unternehmen (14\%) von einem angespannt bis aggressivfeindseligen Prüfungsklima. ${ }^{5}$

Ein Grund, insbesondere für das sachlich/neutrale Betriebsprüfungsklima, dürfte in der zunehmenden fachlich spezialisierten Ausbildung der Betriebsprüfer zu sehen sein. Zwei Drittel der international aufgestellten Unternehmen berichteten, dass in ihrer Betriebsprüfung entweder ein Bundesprüfer oder ein Fachprüfer für Auslandsbeziehungen eingebunden wurde.

Im Gegensatz zu den Jahren unmittelbar nach Einführung der Dokumentationsvorschriften haben Fachprüfer jetzt Zugang zu den relevanten Unternehmensdatenbanken wie Amadeus oder ORBIS und besitzen genügend Erfahrung im Umgang mit diesen Datenbanken. Gegenstand der Betriebsprüfung ist daher immer häufiger die technische Reproduzierbarkeit von Datenbankstudien und die Qualität ausgewählter Vergleichsunternehmen. Bereits heute begründen Betriebsprüfer regelmäßig durch eigene Datenbankstudien höhere Margen deutscher Tochterunternehmen ausländischer Konzernmütter bzw. niedrigere Gewinne von Töchtern deutscher Konzerne im Ausland.

Ein weiterer Grund für die zunehmende Versachlichung der Diskussion mit Betriebsprüfern sind die mittlerweile zahlreicheren internen Schulungen der Finanzverwaltung. Deren Schwerpunkte sind (i) die Verwertbarkeit der Sachverhalts- und Angemessenheitsdokumentation, (ii) die Charakterisierung von Unternehmensarten und die Auswahl von Verrechnungspreismethoden sowie (iii) die Identifizierung von Verrechnungspreisrisiken und Prüfungsansätzen. Es ist zu erwarten, dass diese Themen verstärkt in zukünftigen Betriebsprüfungen behandelt werden.

Wenngleich weiterhin erhebliche Unterschiede in der Behandlung von Verrechnungspreisthemen in der Betriebsprüfung zu beobachten sind, zeichnet sich insgesamt ein Trend

\footnotetext{
${ }^{5}$ Vgl. PwC, Betriebsprüfung 2015, S. 16.
} 
zu einer detaillierten Analyse von Verrechnungspreisthemen im Rahmen von Betriebsprüfungen ab. Die zunehmende Spezialisierung der Betriebsprüfer auf Verrechnungspreisfragen, die zunehmende Komplexität der grenzüberschreitenden Transaktionen zwischen verbundenen Unternehmen sowie das mittlerweile ausgefeilte Regelwerk auf nationaler und internationaler Ebene tragen in gleichem Maße hierzu bei. Steuerpflichtige, die diesem Trend nicht Rechnung tragen, sehen sich in Zukunft verstärkt dem Risiko einer angespannten oder in manchen Fällen sogar aggressiven Prüfungsatmosphäre ausgesetzt. ${ }^{6}$

Aus der PwC Betriebsprüfungsstudie geht ebenfalls hervor, dass am Ende der wenigsten Betriebsprüfungsfälle Finanzgerichtsverfahren oder Verständigungsverfahren stehen. In den meisten Fällen (89\%) wird ein Kompromiss mit der Betriebsprüfung eingegangen, auch wenn dieser zu einer Doppelbesteuerung führt und wahrscheinlich für die Unternehmen vergleichsweise teuer ist. Dies deutet darauf hin, dass bislang die verfügbaren Konfliktlösungsmechanismen von vielen Steuerpflichtigen in den meisten Fällen nicht als effiziente Möglichkeit zur Beseitigung der Doppelbesteuerung gesehen werden. Dies verwundert nicht angesichts der langen Verfahrenszeiten und zeigt die Dringlichkeit hinsichtlich einer Erhöhung der Effizienz von Verständigungs- bzw. Schiedsverfahren.

\subsection{Verwertbarkeit von Dokumentationen/Einhaltung von Vorlagefristen}

Die Frage, ob eine Verrechnungspreisdokumentation verspätet abgegeben worden ist, hängt häufig eng mit der Frage nach der grundsätzlichen Verwertbarkeit einer Dokumentation zusammen. Denn das Ausmaß der Verspätung ist davon abhängig, ob überhaupt eine verwertbare Dokumentation erstellt worden ist. Insofern wird im Folgenden zunächst erörtert, wie die Finanzverwaltung in Betriebsprüfungen die Unverwertbarkeit der Dokumentation begründet. Darüber hinaus werden mögliche Verteidigungsstrategien gegenüber den von der Finanzverwaltung vorgetragenen Argumenten vorgestellt. In einem zweiten Schritt werden dann kurz Probleme im Zusammenhang mit der verspäteten Abgabe einer Verrechnungspreisdokumentation behandelt.

\subsubsection{Verwertbarkeit einer Verrechnungspreisdokumentation}

Wenngleich die rechtlichen Hürden für die Qualifizierung einer Dokumentation vom Gesetzgeber als unverwertbar hoch gesteckt worden sind (vgl. Kap. 2.4), wird bei Betriebsprüfungen von der Finanzverwaltung unerwartet häufig die Unverwertbarkeit einer vorgelegten Dokumentation unterstellt. Hierfür können zum einen weniger sachliche, sondern strategische Motive ausschlaggebend sein, denn bei Vorliegen einer unverwertbaren Dokumentation erhält die Finanzverwaltung weitreichende Schätzbefugnisse

${ }^{6}$ Vgl. PwC, Betriebsprüfung 2015, S. 42. 
(vgl. Kap. 2.4.4). Zum Teil wird die Annahme der Unverwertbarkeit aber auch mit inhaltlichen Argumenten begründet. Hierzu zählen unter anderem:

- Die Dokumentation wurde in englischer Sprache und nicht auf Deutsch eingereicht.

- Die Reproduktion von Datenbankanalysen war nicht möglich.

- Die verwendeten Quellen wurden schlecht dokumentiert.

- In der Dokumentation wurden Transaktionen nicht sachgerecht aggregiert.

- Der Prozess der Preissetzung wurde nicht hinreichend detailliert dokumentiert.

- Die Funktionen und Risiken wurden nicht hinreichend detailliert beschrieben.

- Der Dokumentation fehlt eine quantitative Wertschöpfungsanalyse.

- Die Transaktionsbezogene Nettomargenmethode ist nicht anwendbar, weil die BP das entsprechende Unternehmen nicht als Routineunternehmen qualifiziert.

Ein Teil der oben aufgeführten Argumente bzw. der Probleme, auf die die Argumente abzielen, lässt sich im Vorfeld oder zu Beginn einer Betriebsprüfung relativ leicht lösen.

Spätestens bei Anforderung der Dokumentation durch die Betriebsprüfung sollte ein Antrag bei der Finanzbehörde gestellt werden, Aufzeichnungen in englischer Sprache einreichen zu dürfen. Gemäß Tz. 3.4.16 der VWGV ist das Veranlagungsfinanzamt verpflichtet, unverzüglich über den Antrag zu entscheiden. Wird dem Antrag nicht stattgegeben, weil z. B. nach $\S 2$ Abs. 5 GAufzV Aufzeichnungen grundsätzlich in deutscher Sprache zu erstellen sind, bietet es sich für den Steuerpflichtigen an, der Betriebsprüfung entweder die Übersetzung relevanter Passagen der Dokumentation oder deren Zusammenfassung anzubieten. Oft kann ein für den Steuerpflichtigen akzeptabler Kompromiss gefunden werden, weil Tz. 3.4.16 und 3.2.5. VWGV ausdrücklich feststellen, dass bei Anforderungen von Übersetzungen der Grundsatz der Verhältnismäßigkeit zu beachten ist und nur in begründeten Fällen die Vorlage einer beglaubigten Übersetzung verlangt werden kann.

Dass die Analyseergebnisse nachvollziehbar sein sollten, ist unstrittig. Die Reproduzierbarkeit der Dokumentationen sollte daher bereits bei der Erstellung der Dokumentation sichergestellt werden. Dies gilt für die Ergebnisse von Datenbankstudien ebenso wie die Verwendung von Daten des internen Rechnungswesens (z. B. bei der Verwendung der Kostenaufschlagsmethode), der Verwendung von Plandaten oder von Vergleichspreisen. Strittig dagegen ist, in welchem Umfang die zur Reproduzierbarkeit erforderlichen Unterlagen bereits Teil der Verrechnungspreisdokumentation sein müssen (sodass deren Fehlen die Unverwertbarkeit der Dokumentation verursacht) oder ob sie erst auf Anfrage der Betriebsprüfung im Zuge der allgemeinen Mitwirkungspflichten gemäß $§ 200$ Abs. 1 AO nachzureichen sind. Aufgrund des klar formulierten Zwecks einer Verrechnungspreisdokumentation, nämlich einem sachverständigen Dritten innerhalb angemessener Zeit die Feststellung und Prüfung zu ermöglichen, welche Sachverhalte vom Steuerpflichtigen verwirklicht wurden und ob und inwieweit der Steuerpflichtige dabei den Fremdvergleichsgrundsatz beachtet hat (vgl. VWGV Tz. 3.4.19 und Kap. 2.1), dürfte in der Regel Letzteres der Fall sein. Darüber hinaus klassifizieren die VWGV Tz 3.4.14 bestimmte Dokumente 
als zusätzlich nützliche Informationen, die der Steuerpflichtige vorlegen kann, um seine Aufzeichnungen zu stützen. Hierzu gehören:

- vorliegende Verprobungsrechnungen

- Informationen über Entscheidungskompetenzen

- Darstellung des Ablaufs und des Zeitraums von Preisverhandlungen, Schriftverkehr mit Darstellung konträrer Verhandlungspositionen

- Arbeitnehmerorganigramm und Arbeitsplatzbeschreibungen

- Abhängigkeitsbericht des Vorstands über die Beziehungen zu verbundenen Unternehmen nach $\S 313$ Aktiengesetz.

Von daher ist es fraglich, ob die Nichtvorlage dieser Informationen im Rahmen einer Dokumentation zu deren Unverwertbarkeit führen kann.

In Verrechnungspreisdokumentationen wird häufig die Fremdüblichkeit einer Vielzahl von Verrechnungspreisen eines Stroms von gleichartigen Transaktionen durch die Analyse eines einzigen Gewinnindikators, z. B. der Umsatzrendite einer Vertriebsgesellschaft, durchgeführt. Die ökonomische Analyse einzelner Transaktionen findet häufig nicht statt. Darüber hinaus wird im Rahmen der Sachverhaltsdarstellung, z. B. bei der Beschreibung relevanter Funktionen und Risiken sowie der Preissetzung aus Praktikabilitätserwägungen nicht zwischen einzelnen Transaktionen unterschieden. In der Praxis wird von der BP diese fehlende Transaktionsbezogenheit als Grund für die Unverwertbarkeit einer Dokumentation angeführt.

Diese Argumentation ist aus mehreren Gründen häufig nicht sachgerecht. Erstens hat die Verrechnungspreisdokumentation ein in den VWGV Tz. 3.4.19 klar umrissenes und beschränktes Ziel, nämlich einem Sachverständigen Dritten zu ermöglichen, sich in den Sachverhalt einzuarbeiten. Gerade für die Einarbeitung in einen Sachverhalt ist jedoch eine fein verästelte Darstellung von Funktions- und Risikoprofilen häufig nicht förderlich, sondern der zwangsläufig aggregierende Überblick über Transaktionsströme. Zweitens gestatten sowohl deutsche als auch OECD-RL prinzipiell die Aggregation von Transaktionen (vgl. Kap. 4 und 5.2.5).

Analog hierzu kann auch die unterstellte Unverwertbarkeit aufgrund eines mangelnden Detaillierungsgrades der dargestellten Preissetzung abgelehnt werden. Darüber hinaus ist in Bezug auf die Preissetzung zu beachten, dass es steuerlich unerheblich ist, wie ein Konzern seine Verrechnungspreise für grenzüberschreitende Transaktionen mit verbundenen Unternehmen festlegt. Allein entscheidend ist, ob die Verrechnungspreise dem Fremdvergleichsgrundsatz entsprechen. So hat der BFH bereits in seinem Urteil vom 17.10.2001 entschieden, dass vereinbarte Preise fremdüblich sein können, ,auch wenn der Steuerpflichtige den vereinbarten Preis nach überhaupt keiner Methode, nach keiner anerkannten Standardmethode oder nach der ,falschen“ Methode ermittelt haben sollte“.7

${ }^{7}$ Siehe BFH, Urteil vom 17.10.2001, Az. I R 103/00, (DStR2001), S. 2152. 
Insofern ist zu fragen, wieso eine wenig detaillierte Beschreibung der Preissetzung zu einer unverwertbaren Dokumentation führen kann.

Der Steuerpflichtige muss auch nicht die „objektive Richtigkeit“ der Verrechnungspreismethode begründen, denn die Beweislast liegt im Rahmen einer Betriebsprüfung zunächst bei der Finanzverwaltung. Der Steuerpflichtige muss in seinen Aufzeichnungen nur begründen, warum er subjektiv der Meinung ist, fremdübliche Verrechnungspreise vereinbart zu haben. Insofern ist der Steuerpflichtige frei in seiner Methodenwahl, mit der er die Fremdüblichkeit überprüft. Die Wahl einer ungeeigneten oder gar keiner Methode kann nicht die Unverwertbarkeit der Aufzeichnungen begründen. ${ }^{8}$ Eine plausible Begründung der Fremdüblichkeit anhand von Fremdvergleichsdaten genügt. ${ }^{9}$

Hinsichtlich der Notwendigkeit, eine quantitative Wertschöpfungskette im Rahmen einer Dokumentation zu erstellen, siehe Kap. 4 (Funktions- und Risikoanalyse).

\subsubsection{Verspätete Abgabe einer Verrechnungspreisdokumentation}

In der Praxis tritt das Problem, dass die Betriebsprüfung einen Verspätungszuschlag gemäß $\S 162$ Abs. 4 AO festsetzt, in zwei Fällen auf. Entweder hat der Steuerpflichtige zum Ablauf der Frist von 30 bzw. 60 Tagen (vgl. Kap. 2) sowie unter der Berücksichtigung einer Fristverlängerung keine Dokumentation vorgelegt, oder die zum Ablauf der Frist vorgelegte Dokumentation wird von der Betriebsprüfung als unverwertbar qualifiziert. In beiden Fällen liegt der Finanzverwaltung jedoch ab einem bestimmten Zeitpunkt eine verwertbare Dokumentation unstrittig vor.

In der Praxis können beide Fälle zu erheblichen Verspätungszuschlägen führen, wie folgendes, aus der Praxis stammende, Beispiel illustriert.

\section{Beispiel 6.1: Verspätungszuschläge}

Die Vertriebsgesellschaft VG GmbH vertreibt in Deutschland elektronische und feinmechanische Produkte, die sie von ihrer Mutter aus Japan bezieht, an Großhändler vornehmlich in Südeuropa. Diese veräußern die Produkte an Einzelhändler in den jeweiligen Märkten. Die VG GmbH erzielt durch den Vertrieb eine unstrittig fremdunübliche Umsatzrendite. Im Zuge der Umstellung ihres Geschäftsmodells vertreibt die VG GmbH ab 2007 vornehmlich an Einzelhändler und übernimmt in dem neuen Geschäftsmodell wesentlich mehr Funktionen und Risiken, die unstrittig eine geringfügig höhere fremdübliche Umsatzrendite zur Folge haben müssten. Die Aktualisierung der Verrechnungspreisdokumentation für die Geschäftsjahre 2007-2010 berücksichtigt die

\footnotetext{
${ }^{8}$ Vgl. Wassermeyer, Außensteuerrecht Kommentar § 1 AStG, Anm. 823.30.

${ }^{9}$ Schreiber, VWGV Anm. 212. Vgl. auch Schoppe und Stumpf (2014) für eine ausführliche Diskussion, wann eine Dokumentation unverwertbar ist.
} 
Umstellung des Geschäftsmodells nicht. Allein die Finanzdaten der Benchmarkingstudie werden aktualisiert und der Umsatzrendite der VG GmbH gegenübergestellt.

In der Betriebsprüfung der Jahre 2007-2010 wird die Verrechnungspreisdokumentation der Betriebsprüfung fristgerecht vorgelegt. Im Verlauf der Betriebsprüfung fällt der VG GmbH die fehlerhafte Sachverhaltsdarstellung auf und wird 6 Monate nach Ablauf der Vorlagefrist korrigiert. Die Finanzverwaltung stellt die Verwertbarkeit der korrigierten Dokumentation fest, qualifiziert die ursprünglich vorgelegte Dokumentation aber als unverwertbar. Zum Ende der Betriebsprüfung setzt die Finanzverwaltung einen Verspätungszuschlag von $72.000 €$ fest. Sie beruft sich bei der folgenden Rechnung auf die VWGV Tz. 4.6.3 d.

Fristüberschreitung 180 Tage $(6$ Monate * 30 Tage) * 4 Veranlagungszeiträume $(2007-2010) * 100 € /$ Tag $=72.000 €$

Auf die Festsetzung eines getrennten Verspätungszuschlags für elektronische Produkte, d. h. die Festsetzung eines transaktionsbezogenen Verspätungszuschlags, verzichtet die Finanzverwaltung, weil sie die Zusammenfassung des Vertriebs von elektronischen und feinmechanischen Produkten akzeptiert. Die Verwendung des Mindestverspätungszuschlags von $100 €$ pro Tag wird durch das geringe Verschulden der VG $\mathrm{GmbH}$ und die nur geringfügigen von der VG GmbH gezogenen Vorteile berücksichtigt.

Wenngleich in Bezug auf das geschilderte Beispiel durchaus zu fragen ist, ob die ursprünglich vorgelegte Dokumentation überhaupt unverwertbar ist, zeigt es doch sehr deutlich auf, dass Verspätungszuschläge auch für relativ kurze Fristüberschreitungen eine substantielle Höhe erreichen können. Wesentliche Korrekturen in der Sachverhaltsdarstellung wie zum Beispiel die nachträgliche Dokumentation von Transaktionen sind in der Betriebsprüfung soweit wie möglich zu vermeiden, indem von Anfang an die geforderten Informationen in der Dokumentation zur Verfügung gestellt werden. Darüber hinaus ist zu beachten, dass der Verspätungszuschlag unabhängig von der in der Betriebsprüfung ggf. festgestellten Einkommenskorrektur festgesetzt wird (vgl. VWGV Tz. 4.6.3.). Auch wenn sich im Nachhinein die vereinbarten Verrechnungspreise als fremdüblich herausstellen, droht demnach bei der verspäteten Dokumentationsvorlage ein Verspätungszuschlag.

Ein weiterer Aspekt ist, dass Verspätungszuschläge nicht Gegenstand eines Verständigungsverfahrens sind. Sie können also im Gegensatz zur Doppelbesteuerung nicht im Rahmen von Verständigungsverfahren ausgeräumt werden.

Insgesamt bleibt für die Praxis daher festzuhalten, dass grundsätzliche Diskussionen um die Verwertbarkeit einer Dokumentation und - z. T. damit zusammenhängend - die Festsetzung von Verspätungszuschlägen einen erheblichen Raum in der Betriebsprüfung einnehmen können und die eigentliche Diskussion um die Fremdüblichkeit von Verrechnungspreisen überschatten können. Eine entsprechende Diskussion sollte von den Steuerpflichtigen nach Möglichkeit durch die vollständige und fristgerechte Vorlage einer Dokumentation vermieden werden. 


\subsection{Auswahl der Verrechnungspreismethode (Finanzverwaltung vs. Praxis)}

Im Rahmen der Angemessenheitsanalyse ist eine Verrechnungspreismethode zu wählen, mit der der Fremdvergleich geführt und dokumentiert wird. Eine mögliche Methode ist die Transaktionsbezogene Nettomargenmethode (,TNMM“), die auf einer Suche nach Vergleichsunternehmen in Datenbanken basiert, welche die Nettomargen vergleichbarer externer Transaktionen ermittelt. Diese Studien werden häufig aus folgenden Gründen in Betriebsprüfungen aufgegriffen:

- Die Vergleichbarkeit der Produkte und Branchen wird angezweifelt.

- Das untersuchte verbundene Unternehmen wird als sog. Mittelunternehmen qualifiziert, für welches die Anwendung von Suchen nach Vergleichsunternehmen in Datenbanken abzulehnen ist.

- Es wird unterstellt, dass ein Routineunternehmen nur Geschäftstätigkeiten ohne jegliche Risiken ausüben darf und somit die TNMM bei Vorliegen z. B. allgemeiner Marktrisiken nicht angewendet werden kann.

- Die Betriebsprüfung lehnt die Anwendung von Suchen nach Vergleichsunternehmen in Datenbanken generell ab.

Eine freie Schätzung der Besteuerungsgrundlagen durch die Finanzverwaltung ist unzulässig. Hat der Steuerpflichtige seine Mitwirkungspflichten nach $\S 90$ Abs. 3 AO erfüllt, so hat grundsätzlich die Finanzverwaltung den Nachweis zu erbringen, dass die Verrechnungspreise nicht dem Fremdvergleichsgrundsatz entsprechen, vgl. Kap. 2.4.1. ${ }^{10}$ Bei Nichtvorlage einer Verrechnungspreisdokumentation oder bei Vorlage unverwertbarer Aufzeichnungen kann die Finanzbehörde eine Schätzung vornehmen und, falls die Einkünfte dabei nur innerhalb eines bestimmten Rahmens geschätzt werden können, diesen Rahmen zulasten des Steuerpflichtigen vollständig ausschöpfen. ${ }^{11}$ Diese Schätzung hat auf dem Steuerpflichtigen zugänglichen Daten zu beruhen und muss empirisch nachvollzogen werden können. ${ }^{12}$

Des Weiteren kann die Verteidigung der angewendeten Suche nach Vergleichsunternehmen in Datenbanken auf folgende Argumente gestützt werden:

- Sollte die Betriebsprüfung die Suche nach Vergleichsunternehmen in Datenbanken aus dem Grund ablehnen, dass diese auf pan-europäischen Datenbanken beruht, so kann darauf hingewiesen werden, dass fundierte Untersuchungen vorliegen, welche die Verwendung pan-europäischer Daten unterstützen. ${ }^{13}$ Diese Untersuchungen haben gezeigt, dass die europäischen Märkte als hinreichend vergleichbar angesehen werden können,

\footnotetext{
${ }^{10} \mathrm{Vgl}$. Tz. 4.2 VWGV.

${ }^{11}$ Vgl. § 162 Abs. 3 Satz 2 AO.

12 Vgl. Tz. 4.5 VWGV.

${ }^{13}$ Z. B. Europäische Kommission, EU Joint Transfer Pricing Forum (2004), Is Europe One Market.
} 
so dass die Vergleichsunternehmen auch aus anderen europäischen Ländern als dem des untersuchten Unternehmens stammen können. Folglich kann die Suche nach Vergleichsunternehmen in Datenbanken nicht nur auf nationalen, sondern auch auf paneuropäischen Datenbanken beruhen. ${ }^{14}$

- Häufig lehnt die Betriebsprüfung die Anwendung der TNMM aus dem Grund ab, dass das untersuchte Unternehmen nicht als Routineunternehmen einzustufen sei. Als Begründung wird dabei vorgebracht, dass ein Routineunternehmen keine Risiken übernehmen dürfe. Ein Unternehmen wird jedoch in der Regel dann als Routineunternehmen qualifiziert, wenn es über keine wesentlichen immateriellen Wirtschaftsgüter verfügt. Die Ausübung vollständig risikofreier Tätigkeiten ist dagegen nicht erforderlich und wird kaum beobachtbar sein. Damit ist die Anwendung von Methoden, die auf einer Suche nach Vergleichsunternehmen in Datenbanken beruhen, in der Regel dann möglich, wenn das Unternehmen ein Funktions- und Risikoprofil aufweist, für das zunächst eingeschränkt vergleichbare Unternehmen gefunden werden können. Dies ist zumeist dann der Fall, wenn das Unternehmen über keine wesentlichen wertbestimmenden immateriellen Wirtschaftsgüter verfügt.

- Zuletzt ist darauf hinzuweisen, dass ein externer Vergleich mittels einer Suche nach Vergleichsunternehmen in Datenbanken im Einklang mit den OECD-RL und der Methodenhierarchie i. S. d. $\S 1$ Abs. 3 AStG steht. Die Betriebsprüfung kann somit die Anwendung der TNMM nicht grundsätzlich ablehnen.

- Um Diskussionen in der Betriebsprüfung zur Anwendbarkeit und der konkreten Anwendung der TNMM bereits frühzeitig zu verhindern, sollte eine möglichst vollständige Verrechnungspreisdokumentation vorgelegt werden, in der die Qualifizierung des Unternehmens als Routineunternehmen im Rahmen der Funktions- und Risikoanalyse dargestellt und die Vergleichbarkeit der unverbundenen Unternehmen aufgezeigt wird.

\subsubsection{Fall 1- Standardmethoden vs. gewinnorientierte Methoden}

Die Diskussionen mit der Betriebsprüfung hinsichtlich der Auswahl einer geeigneten Verrechnungspreismethode sollen an folgendem Fall verdeutlicht werden. Der Sachverhalt stellt sich dabei wie folgt dar:

- Ein Automobilkonzern mit Konzernmutter im Ausland vertreibt LKWs über verbundene Händler und unverbundene Vertragshändler in Deutschland. Zwischen den verbundenen und unverbundenen Händlern liegen keine systematischen Unterschiede vor

\footnotetext{
${ }^{14}$ Zwar hat die OECD im BEPS-Papier zur Dokumentation eine Präferenz für lokale Vergleichsunternehmen geäußert; vgl. OECD (2015), Transfer Pricing Documentation and Country-by-Country Reporting, S. 20. Allerdings gilt dies im Einklang mit den OECD RL nicht zwingend für hochintegrierte Märkte wie die EU. Zumal die EU derzeit die Anwendbarkeit Pan-Europäischer Vergleichsstudien im EU Joint Transfer Pricing Forum überprüft und dabei davon ausgeht, dass es sich dabei um einen wohletablierten Best-Practice Ansatz handelt; vgl. EU JTPF 2016 Working Paper on Comparables in th EU.
} 
(z. B. Standort, Größe des Betriebs- und die Produktsegmente betreffend). Die LKWs werden zu gleichen Preisen an die verbundenen und unverbundenen Händler geliefert.

- Im Betriebsprüfungszeitraum erzielen die verbundenen Händler Verluste.

- Der Steuerpflichtige wendet die Preisvergleichsmethode zur Preissetzung an und die Brutto-Kostenaufschlagsmethode, um die Fremdüblichkeit der Verrechnungspreise nachzuweisen. Zwar ist aufgrund unterschiedlicher Ausstattungsmerkmale der LKWs, die den Preis beeinflussen, ein direkter Preisvergleich nicht möglich, doch kann ermittelt werden, mit welchen Kostenaufschlägen die Konzernmutter die LKWs an die unverbundenen Händler vertreibt. Daraus wird eine Bandbreite an Kostenaufschlägen ermittelt, in der die Kostenaufschläge an die verbundenen Händler liegen.

Die Betriebsprüfung greift die Verrechnungspreise aufgrund der Verlustsituation beim deutschen Steuerpflichtigen auf und akzeptiert die Kostenaufschlagsmethode zur Verprobung der Fremdüblichkeit der Verrechnungspreise nicht. Die Begründung hierfür lautet, dass die unverbundenen und verbundenen Händler nicht uneingeschränkt vergleichbar sind. Zudem wären die Gewinne aufgrund weiterer Faktoren, wie bspw. Händlerboni, neben den Einkaufspreisen nicht vergleichbar.

Grundsätzlich sind gem. § 1 Abs. 3 Satz 1 AStG die Verrechnungspreise vorrangig nach den Standardmethoden zu ermitteln. Auch nach Auffassung der OECD sollte den Standardmethoden der Vorzug gegeben werden, wenn vergleichbare Fremdgeschäftsvorfälle vorliegen und sowohl eine Standardmethode als auch eine Gewinnmethode gleichermaßen zuverlässig angewendet werden können (vgl. Tz. 2.3 OECD-RL). Da der Steuerpflichtige nachweisen kann, dass die Transaktionen hinreichend vergleichbar sind, sind die Standardmethoden im vorliegenden Fall somit vorrangig anzuwenden. Um das Ergebnis aus der Anwendung der Standardmethode zu substantiieren, sieht die abschließende Einigung mit der Betriebsprüfung schließlich vor, dass die Preise für die LKWs zwischen der ausländischen Konzernmutter und den verbundenen Händlern in Deutschland zusätzlich auf Grundlage der TNMM und einer Suche nach Vergleichsunternehmen in Datenbanken verprobt werden sollen. Anschließend wird im Rahmen des Kompromisses eine Gewinnkorrektur auf den unteren Bereich der Bandbreite, die durch die Suche nach Vergleichsunternehmen ermittelt worden ist, durchgeführt.

Fazit: Zwar sind die Verrechnungspreise grundsätzlich vorrangig nach den Standardmethoden zu ermitteln, doch kann in manchen Betriebsprüfungsfällen auch eine zusätzliche Verprobung anhand gewinnorientierter Methoden zielführend sein.

\subsubsection{Fall 2- Profit Split vs. Preisvergleich}

Ein weiterer Fall, der die aktuelle Diskussion hinsichtlich der Auswahl einer geeigneten Verrechnungspreismethode mit der Betriebsprüfung verdeutlichen soll, stellt sich wie folgt dar: 
- Ein deutscher Finanzdienstleister erbringt Produktionsleistungen (Konzipierung sowie laufende Anpassung von Finanzprodukten) und Vertriebsdienstleistungen an eine ausländische Konzerngesellschaft.

- Die Vergütung der Produktionsleistung erfolgt im Betriebsprüfungszeitraum anhand eines externen Preisvergleichs auf Basis einer Marktstudie. Das Ergebnis der Studie führt zu einer sehr hohen Vergütung der Produktionsleistung (Gewinnaufschlag von 400\% auf die Kosten). Die Vertriebstätigkeit wird auf Kostenbasis an die ausländische Konzerngesellschaft weiterbelastet. Der Residualgewinn verbleibt damit bei der ausländischen Gesellschaft.

- Für die Produktionsleistung wird eine umfangreiche Verrechnungspreisdokumentation erstellt, einschließlich einer Analyse der Wertschöpfungskette. Die bisherige Verrechnung auf Basis des Preisvergleichs wird genau analysiert und aufgrund fehlender direkt vergleichbarer Preise und entsprechender Unzuverlässigkeit der Ergebnisse verworfen. Aus diesem Grund werden die zukünftigen Verrechnungspreise für Produktionsleistungen auf Basis der Kostenaufschlagsmethode und den Ergebnissen einer Suche nach Vergleichsunternehmen in Datenbanken ermittelt.

Der Betriebsprüfung wird die Verrechnungspreisstudie für die zukünftige Verrechnung der Produktionsleistung anhand der Kostenaufschlagsmethode vorgelegt. Diese stellt jedoch sowohl die Verrechnung auf Basis des Preisvergleichs als auch auf Basis der Kostenaufschlagsmethode infrage und präsentiert eigene Berechnungen, basierend auf einem Profit Split Modell. Dabei wird der Gewinn entsprechend dem jeweiligen Personalaufwand zwischen dem deutschen Finanzdienstleister und der ausländischen Konzerngesellschaft geteilt. Des Weiteren befindet die Betriebsprüfung die an die ausländische Konzerngesellschaft in Rechnung gestellten Kosten für die Vertriebsdienstleistungen als zu gering.

Als Verteidigungsstrategie wird vom Steuerpflichtigen argumentiert, dass die Kostenaufschlagsmethode eine zuverlässig anwendbare Methode ist. Dies kann auch anhand interner Daten belegt werden, da vergleichbare Produktionsdienstleistungen auch an unverbundene Dritte erbracht werden und hierfür eine Bandbreite an Kostenaufschlägen ermittelt werden kann. Das von der Betriebsprüfung vorgelegte Profit Split Modell, insbesondere die Gewinnverteilung auf Basis des Personalaufwands, reflektiert hingegen nicht die Verteilung der Werttreiber sowie der Funktionen und Risiken und stellt somit im vorliegenden Fall keine angemessene Verrechnungspreismethode dar. In der letztendlich mit der Betriebsprüfung erzielten Einigung verständigen sich beide Parteien darauf, dass die Kostenaufschlagsmethode sowohl für die Vertriebsdienstleistungen als auch für die Produktionsleistungen angewendet werden kann, wodurch sich die Verrechnungspreise für den Vertrieb erhöhen und für die Produktion reduzieren.

Fazit: Die Profit Split Methode kann in dem vorliegenden Fall als gewinnorientierte Methode nur nachrangig nach den Standardmethoden angewendet werden. ${ }^{15} \mathrm{Da}$ im vorliegenden Fall die Vergleichbarkeit der Fremdvergleichswerte für die Kostenaufschlagsmethode vom Steuerpflichtigen nachgewiesen werden kann, muss die Finanzverwaltung

${ }_{15} \S 1$ Abs. 3 Satz 1 AStG. 
diese Methode akzeptieren. Die Profit Split Methode gewinnt im Zuge des BEPS-Projekts der OECD jedoch weiter an Bedeutung und soll nach Auffassung der OECD in Zukunft häufiger zur Bestimmung angemessener Verrechnungspreise eingesetzt werden.

Die Auswahl der Verrechnungspreismethode und deren Anerkennung durch die Betriebsprüfung ist somit ein kritischer und entscheidender Aspekt in der Verrechnungspreispraxis. Im Fall einer Betriebsprüfung sind nicht nur gute Argumente für die Auswahl einer bestimmten Methode zu finden. Es sind häufig auch Gründe vorzulegen, weshalb andere Methoden im jeweiligen Fall nicht anwendbar sind.

\subsection{Häufige Prüfungsfelder}

Im Folgenden werden spezielle und häufig vorkommende Themenbereiche in aktuellen Betriebsprüfungen kurz dargestellt und anhand einzelner Fälle näher erläutert. Dabei wird auch darauf eingegangen, was im jeweiligen Fall eine geeignete Verteidigungsstrategie sein kann.

\subsubsection{Lizenzen}

Neben der Frage der Fremdüblichkeit einer Lizenz dem Grunde nach führt insbesondere auch die Höhe der Lizenzgebühr häufig zu Diskussionen in der Betriebsprüfung. Oft werden Lizenzgebühren ganz oder teilweise in den Fällen nicht akzeptiert, in denen sie zu Verlusten im Inland oder hohen Gewinnen in Ausland führen. Auch bei einer Umstellung der Lizenzgebühren (z. B. hinsichtlich der Höhe des Lizenzsatzes oder der Berechnungsmethode) kommt es häufig zu Diskussionen mit der Betriebsprüfung, da diese die wirtschaftlichen Gründe für die Umstellung anzweifelt.

\section{Beispiel 6.2: Lizenzgebühren}

An folgendem Fall wird eine mögliche Diskussion mit der Betriebsprüfung hinsichtlich Lizenzzahlungen veranschaulicht:

- Ein ausländischer Konzern mit mehreren Tochtergesellschaften in Deutschland betreibt ein B2B-Geschäft. Die ausländische Muttergesellschaft ist für alle markenentwickelnden und -stärkenden Aktivitäten sowie die markenbezogene Öffentlichkeitsarbeit zuständig und trägt die entsprechenden Kosten. Die deutsche Tochtergesellschaft zahlt an die Muttergesellschaft im Ausland eine Markenlizenzgebühr auf Umsatzbasis.

- Der Betriebsprüfung wird eine Verrechnungspreisdokumentation vorgelegt, in der die Bedeutung der Marke und der Aktivitäten der Muttergesellschaft sowie der Nutzen der Lizenznehmer aus der Lizenzierung beschrieben werden. Für die Angemessenheitsanalyse der Markenlizenz wird nach vergleichbaren Lizenzvereinbarungen zwischen fremden Dritten in öffentlich zugänglichen Lizenzdatenbanken gesucht. Die Lizenzgebühr wird anschließend am unteren Rand der ermittelten interquartilen 
Bandbreite festgesetzt. Nach Auffassung der Betriebsprüfung in dem beschriebenen Fall sind grundsätzlich keine Fremdvergleichsdaten für immaterielle Wirtschaftsgüter verfügbar, da diese immer einzigartig sind und somit ein Vergleich mit anderen immateriellen Wirtschaftsgütern nicht möglich ist. Stattdessen soll ein hypothetischer Fremdvergleich zur Bestimmung angemessener Lizenzzahlungen angewendet werden.

Als Argumentation gegenüber der Betriebsprüfung wird angeführt, dass die Lizenzsätze aus Verträgen zwischen fremden Dritten zumindest eingeschränkt vergleichbar sind, da der Gegenstand der Lizenzverträge vergleichbar ist und die Lizenzen unter vergleichbaren Bedingungen vergeben wurden. Aus diesem Grund kann eine eingeschränkte Bandbreite ermittelt werden. Da somit zumindest eingeschränkt vergleichbare Werte verfügbar sind, ist der hypothetische Fremdvergleich nicht anwendbar. ${ }^{16}$ Zusätzlich zur Lizenzstudie wird das Ergebnis mithilfe einer überschlägigen Gewinnanteilsberechnung verprobt (sog. Knoppe-Formel). Die Betriebsprüfung akzeptiert daraufhin die Lizenzen auch der Höhe nach. Derzeit bestehen jedoch Tendenzen in der deutschen Finanzverwaltung, die Vergleichbarkeit eines solchen Ansatzes, d. h. der Vergleich mit Lizenzverträgen fremder Dritter, infrage zu stellen und als verlässlichen Ansatz nicht mehr zuzulassen. Zudem sollen nach Überarbeitung der OECD-RL im Rahmen des BEPS-Projekts Daumenregeln wie die Knoppe-Formel nach Auffassung der OECD nicht mehr als Nachweis der Angemessenheit von Lizenzzahlungen herangezogen werden können. ${ }^{17}$ Die Anwendung der Preisvergleichsmethode mit Rückgriff auf vergleichbare Lizenzverträge zwischen fremden Dritten soll zwar weiterhin möglich sein, doch soll der Profit Split Methode zukünftig größere Bedeutung beigemessen werden. Dabei wird wahrscheinlich auch die Fremdüblichkeit von Lizenzraten häufiger auch mit Hilfe der Profit Split Methode oder Bewertungsmethoden bzw. dem hypothetischen Fremdvergleich überprüft werden. Jedenfalls wurden die Vergleichbarkeitsanforderungen für die Anwendung der Preisvergleichsmethode erhöht. Hier sind zahlreiche Kriterien explizit zu prüfen und zu dokumentieren.

Fazit: Die Fremdüblichkeit von Lizenzen für immaterielle Wirtschaftsgüter kann im Prinzip durch Lizenzstudien verprobt werden, sofern zumindest eingeschränkt vergleichbare Daten ermittelt werden können. Die Anforderungen für die Vergleichbarkeit wurden aber erhöht. Nur für den Fall, dass solche Werte nicht ermittelt werden können, ist der hypothetische Fremdvergleich anzuwenden.

\subsubsection{Kostenumlagen und Dienstleistungen}

Häufig werden Kostenumlagen und Dienstleistungen in Betriebsprüfungen aufgegriffen, da ein Nutzennachweis der Dienstleistungen oft nur schwer zu erbringen ist. Zwar ist eine genaue Nutzenanalyse aus den konzerninternen Dienstleistungen grundsätzlich erforderlich, aber in vielen Fällen nicht praktikabel, so dass es empfehlenswert ist,

\footnotetext{
${ }^{16}$ Vgl. § 1 Abs. 3 Satz 5 AStG Umkehrschluss.

${ }^{17}$ Vgl. OECD (2015) Aligning Transfer Pricing Outcomes with Value Creation, S. 93 ff.
} 
unterschiedliche Beispiele für die erbrachten/empfangenen Dienstleistungen zu dokumentieren. Zudem sollten die Kostenbasis und die Ermittlung des Verteilungsschlüssels dargestellt werden können. Auch liegt es im Ermessen der Betriebsprüfung, ggf. die Prüfung auf einzelne Jahre und Sachverhalte zu beschränken, da mit dem Nutzen- und Fremdvergleichsnachweis der Kostenumlagen für jedes einzelne geprüfte Jahr oft ein unverhältnismäßig großer Aufwand für den Steuerpflichtigen verbunden ist.

\section{Beispiel 6.3: Kostenumlage}

Im folgenden Fall wird die Diskussion der Kostenumlage in einer Betriebsprüfung veranschaulicht:

- Die Bottle Inc. ist ein US-amerikanischer Hersteller mit weltweiten Produktionsstätten. In Deutschland vertreibt die Gruppe ihre Produkte durch die D GmbH. Diese wird jährlich mit einer Kostenumlage im Rahmen eines Dienstleistungsvertrags belastet, u. a. für Kosten im Bereich Controlling, Revision, Human Resources, Öffentlichkeitsarbeit etc. Der Gewinnaufschlag beträgt 5\%. Shareholderkosten (z. B. Kosten der Hauptversammlung) werden nicht verrechnet.

- Die Dokumentation der Bottle Inc. umfasst den Kostenumlagevertrag; eine Aufstellung der Kosten, die Gegenstand der Umlage sind; die Kostenschlüssel und Berechnungsgrundlagen; eine abstrakte Beschreibung der erbrachten Dienstleistungen für jede einzelne Kategorie; sowie einzelne Beispiele für den Nutzen bei der Tochtergesellschaft. Die Dokumentation enthält jedoch keine Quantifizierung des Nutzens. Des Weiteren wird der Anteil der Shareholderkosten im Wege der Schätzung bestimmt und nur oberflächlich dokumentiert. Die genaue operative Umsetzung ist zudem nicht Gegenstand des Vertrags.

Die Betriebsprüfung unterstellt in voller Höhe eine verdeckte Gewinnausschüttung, da im Vertrag der D GmbH kein Einsichtsrecht eingeräumt wird und diese die Kosten somit offensichtlich nicht auf Angemessenheit hin überprüfen kann. Ein Gewinnaufschlag sei unzulässig, weil ein Poolvertrag vorliegt. ${ }^{18}$ Zudem handelt es sich nach Auffassung der Betriebsprüfung bei den meisten Kosten um Gesellschafteraufwand; ein Nutzen für die D $\mathrm{GmbH}$ sei nicht nachgewiesen worden. Für andere Dienstleistungen sei zwar ein lokaler Nutzen gegeben, dieser könne jedoch nicht quantifiziert werden. Da auch eine Schätzung nicht möglich sei, liege in voller Höhe eine verdeckte Gewinnausschüttung vor, vgl. Kap. 2.2.3.

Als Verteidigungsstrategie wird vorgebracht, dass das fehlende Einsichtsrecht im Dienstleistungsvertrag ein rein formales Argument darstellt, das steuerlich irrelevant ist. Hinsichtlich des Gewinnaufschlags wird zudem vorgebracht, dass der Steuerpflichtige einen „Service Vertrag“ abgeschlossen hat und keine Einzelverrechnung vorliegt. Der Steuerpflichtige hat in dieser Konstellation somit ein faktisches Wahlrecht zwischen dem

$\overline{18}$ Tz. 2.2 VWG-Umlageverträge. 
Poolvertrag und einem Dienstleistungsvertrag mittels der indirekten Methode. Dieses hat die D GmbH zugunsten des Dienstleistungsvertrags ausgeübt, was von der Betriebsprüfung akzeptiert werden muss.

Fazit: Die Finanzverwaltung hat den Geschäftsvorfall so zu prüfen, wie er vom Steuerpflichtigen tatsächlich durchgeführt und gestaltet wurde. Eine Umqualifizierung wäre in der Regel völlig willkürlich. ${ }^{19}$

\subsubsection{Finanztransaktionen}

Neben Waren- und Dienstleistungstransaktionen geraten auch Finanztransaktionen zunehmend in den Fokus der Betriebsprüfung. Zu Finanztransaktionen zählen z. B. konzerninterne Darlehen, Bürgschaften, Patronatserklärungen und Cash Pools. Dabei wird häufig nicht nur die Angemessenheit der Höhe der Vergütung geprüft, sondern auch die damit zusammenhängenden Konditionen der Finanztransaktion.

\section{Beispiel 6.4}

Finanztransaktionen Folgender Fall veranschaulicht den Stand derzeitiger Diskussionen in Betriebsprüfungen bezüglich konzerninterner Finanztransaktionen:

- Ein deutsches Unternehmen erhält ein Darlehen von einer ausländischen Finanzierungsgesellschaft. Das Darlehensvolumen beträgt ca. 100 Mio. €. Der Darlehensvertrag ist von beiden Seiten jederzeit kündbar. Das Darlehen ist festverzinslich und die Zinsen betragen ca. 7\% für 3-4 Jahre. Im Zeitverlauf sind die Zinsen am Markt jedoch stark gesunken.

- Der Steuerpflichtige legt der Betriebsprüfung eine Verrechnungspreisdokumentation vor, allerdings ohne eine Studie über Darlehen mit vergleichbaren Kündigungsoptionen.

Die Betriebsprüfung zweifelt die Fremdüblichkeit der Konditionen an, da fremde Dritte bei beidseitigem Kündigungsrecht variable Zinsen vereinbart hätten. Aus diesem Grund wird eine Einkünftekorrektur auf Basis einer variablen Verzinsung (EURIBOR $+\mathrm{x} \%$ ) durchgeführt.

Der Steuerpflichtige erstellt daraufhin eine Analyse basierend auf einem Drittvergleich (Suche nach vergleichbaren Unternehmensschuldverschreibungen). Hierfür werden vergleichbare Darlehenstransaktionen mit beidseitigem Kündigungsrecht und fixer Verzinsung gesucht. Die Auswertung zeigt, dass vergleichbare Verträge existieren und dass die konzerninterne Verzinsung innerhalb der ermittelten Bandbreite liegt. Die Ergebnisse des Drittvergleichs bestätigten somit die Fremdüblichkeit der konzernintern vereinbarten Darlehenskonditionen.

${ }^{19}$ Vgl. Tz. 1.64 OECD-RL. 
Fazit: Die Fremdüblichkeit von Zinszahlungen für konzerninterne Darlehen kann häufig durch vergleichbare Unternehmensschuldverschreibungen verprobt werden, was von der Finanzverwaltung zunehmend akzeptiert wird.

\subsubsection{Umstrukturierung/Funktionsverlagerung}

Ab VZ 2008 sind erstmals die Vorschriften zur Funktionsverlagerung i. S. d. § 1 Abs. 3 Satz 9 AStG anwendbar. Die Finanzverwaltung wollte diese auch schon für frühere Veranlagungszeiträume anwenden, was an folgendem Fall veranschaulicht wird.

\section{Beispiel 6.5: Funktionsverlagerung}

- Der Steuerpflichtige ist ein deutscher Produzent eines ausländischen Herstellers von Freizeitartikeln. Die ausländische Muttergesellschaft lizenziert für die Produktion benötigtes IP an den deutschen Produzenten. Die Produktion, einschließlich des Lizenzvertrags, wird in Deutschland 1998 beendet und in Osteuropa fortgesetzt. Der Steuerpflichtige veräußert deshalb Vorräte, Maschinen, technische Anlagen sowie die Betriebs- und Geschäftsausstattung an die osteuropäische Schwestergesellschaft Shareholderkosten, die Lizenznehmerin für das IP wird und die Produktion übernimmt.

Nach Auffassung der Betriebsprüfung handelt es sich hierbei um eine Verlagerung der Produktionsfunktion. Die Vergütung hierfür ist nur für materielle Wirtschaftsgüter, d. h. die Vorräte, Maschinen und Anlagen, gezahlt worden. Voneinander unabhängige Dritte hätten nach Auffassung der Betriebsprüfung eine Vereinbarung über die Vergütung der Schließungskosten und den Verzicht auf das Gewinnpotential des verlagernden Unternehmens unter Berücksichtigung des Gewinnpotentials des übernehmenden Unternehmens getroffen. Ordentliche und gewissenhafte Geschäftsleiter würden eine Funktionsverlagerung als einheitlichen Vorgang beurteilen und das Entgelt für die Verlagerung als Transferpaket auf Basis des hypothetischen Fremdvergleichs bestimmen. Die Betriebsprüfung berechnet aus diesem Grund einen Mindestpreis in zweistelliger Millionenhöhe in Anlehnung an den damaligen Entwurf der VWG-FVerl vom 17.7.2009.

Als Gegenargumentation wird vom Steuerpflichtigen angeführt, dass $\S 1$ Abs. 3 Satz 9 AStG i. V. m. $§ 1$ FVerlV nicht rückwirkend angewendet werden kann. Damit hat die Bewertung der Übertragung der Funktion auch nicht als Ganzes zu erfolgen, d. h., der Steuerpflichtige hat kein Transferpaket zu ermitteln. Die Übertragung der Wirtschaftsgüter und erbrachten Dienstleistungen ist vielmehr auf Basis des Fremdvergleichsgrundsatzes einzeln zu bewerten. Die immateriellen Wirtschaftsgüter für die Produktion stehen im Eigentum der Muttergesellschaft und sind deshalb nicht zu vergüten. Auch die Kundenbasis besteht ausschließlich aus Konzerngesellschaften, da der deutsche Produzent die Sportgeräte nur an verbundene Unternehmen veräußert hat. Damit besteht für den 
Kundenstamm keine Ausgleichspflicht. Zudem besitzt der Steuerpflichtige keine immateriellen Wirtschaftsgüter und auch der Lizenzvertrag mit der ausländischen Muttergesellschaft ist gekündigt worden, so dass die Grundlage für die Produktion weggefallen ist. Damit konnte auch keine Geschäftschance an die osteuropäische Gesellschaft übertragen werden. Folglich ist auch hierfür keine Ausgleichszahlung zu leisten. Die mit der Betriebsprüfung erzielte Einigung führt auf Basis der oben genannten Argumente zu keiner Anpassung.

Fazit: Die Vorschriften zur Funktionsverlagerung sind erstmalig für den VZ 2008 anzuwenden..$^{20}$ Eine rückwirkende Anwendung ist nicht zulässig. Da keine immateriellen Wirtschaftsgüter übertragen werden, wäre im vorliegenden Fall ab VZ 2008 zudem die Escape-Klausel des $\S 1$ Abs. 3 Satz 10 AStG anwendbar, wonach für die Übertragung ausschließlich materieller Wirtschaftsgüter Einzelverrechnungspreise anzusetzen sind.

\subsection{Betriebsprüfungsmanagement}

Als Quintessenz aus zahlreichen Betriebsprüfungsfällen kann folgende Schlussfolgerung gezogen werden:

1. Die Betriebsprüfer sind von Jahr zu Jahr besser gerüstet und lernen ständig dazu. Verrechnungspreise rücken mehr denn je in den Fokus der Betriebsprüfer. Es werden immer mehr spezialisierte und geschulte Betriebsprüfer eingesetzt und der Prüfungsschwerpunkt wird vermehrt auf das Thema Verrechnungspreise gelegt.

2. Die Wahrscheinlichkeit eines Aufgriffs durch die Betriebsprüfung sowie das Anpassungsvolumen steigen stetig.

3. Prüfer versuchen teilweise über eigene Verrechnungspreisansätze - z. B. Datenbankanalysen oder alternative Verrechnungspreisansätze - Einkommensanpassungen zu begründen.

4. Eine solideVerrechnungspreisdokumentationistdiebesteBetriebsprüfungsvorbereitung.

Das in Abb. 6.2 dargestellte Modell verdeutlicht das Risiko hinsichtlich der Verrechnungspreise in einer Betriebsprüfung. Es ist ein guter Ausgangspunkt für eine erfolgreiche Risikobegrenzung und damit die Grundlage für ein erfolgreiches Betriebsprüfungsmanagement.

Die Risiken einer Betriebsprüfung bestehen im Wesentlichen aus drei Arten negativer Konsequenzen, die sich in einer Betriebsprüfung realisieren können:

1. Das Anpassungsrisiko: Dieses realisiert sich durch Einkommensanpassungen, die wiederum zu Mehrsteuern und Zinszahlung führen.

${ }^{20}$ Vgl. § 21 Abs. 16 AStG. 


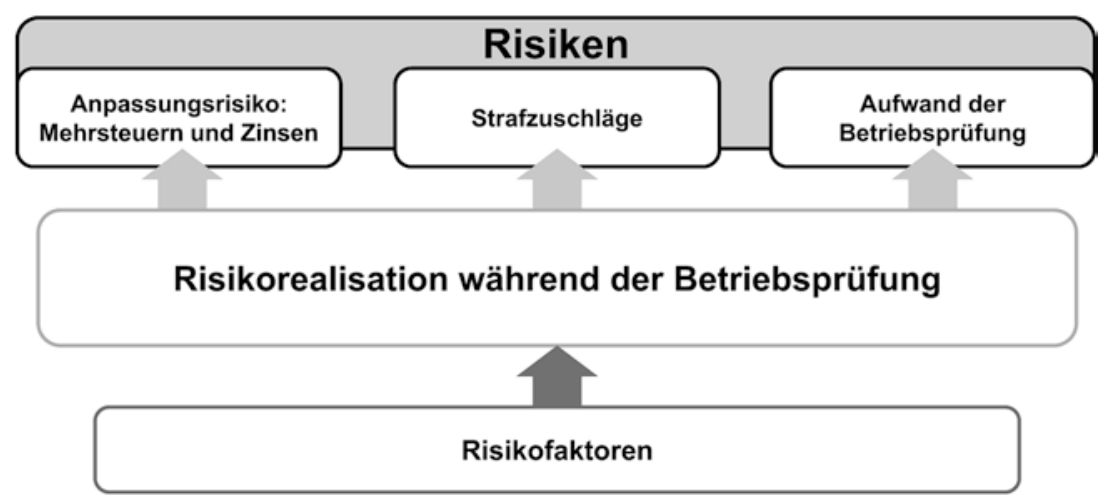

Abb. 6.2 Risiken

2. Strafzuschläge: Diese können für die Nichterfüllung der Dokumentationspflichten auferlegt werden, d. h. für die Vorlage einer nicht verwertbaren Dokumentation oder für die verspätete Vorlage dieser Dokumentation.

3. Aufwand der Betriebsprüfungen: Für die Vorbereitung und das Management der Betriebsprüfungen sind Ressourcen (insbesondere Zeit der Mitarbeiter) erforderlich, um die Anforderungen der Betriebsprüfung zu erfüllen und die Prüfung zu einem akzeptablen Ergebnis zu führen. Diese können von Betriebsprüfungen zu Betriebsprüfungen erheblich abweichen.

Ziel des Steuerpflichtigen ist es, die genannten Risiken möglichst gering zu halten. Anpassungen der Verrechnungspreise bzw. Einkommensanpassungen, die zu mehr Steuern und Zinsen führen, sowie die Auferlegung von Strafzuschlägen sollten möglichst vermieden werden. Gleichzeitig sollte auch der Aufwand der Betriebsprüfung in Grenzen gehalten und eine umfassende, umfangreiche und langwierige Auseinandersetzung mit der Finanzverwaltung vermieden werden.

Die Ziele, Minimierung der Mehrsteuern und Strafzuschläge einerseits, Eingrenzung des Aufwands für und in der Betriebsprüfung andererseits, scheinen auf den ersten Blick im Konflikt zu stehen: Zum Aufbau einer möglichst wenig angreifbaren und verwertbaren Dokumentation, durch die die ersten beiden Ziele besser erreicht werden können, werden unter normalen Umständen mehr Ressourcen benötigt als für eine Dokumentation, die gerade ausreichend ist, um die Schwelle der Verwertbarkeit zu überschreiten. Andererseits kann eine Dokumentation, für die ein gewisser Aufwand betrieben wird, um eine fundierte Fremdvergleichsanalyse zur Verteidigung der Verrechnungspreise zu erstellen, Aufwand in den eigentlichen Auseinandersetzungen mit der Betriebsprüfung ersparen: Je mehr Sachverhaltsfragen durch die Dokumentation geklärt werden und je fundierter die Angemessenheitsanalyse ist, umso weniger Fragen werden die Betriebsprüfer im Verlauf der Betriebsprüfung zu den Verrechnungspreisen stellen und umso weniger wahrscheinlich ist es, dass die Prüfer die Verrechnungspreise grundlegend infrage stellen. 


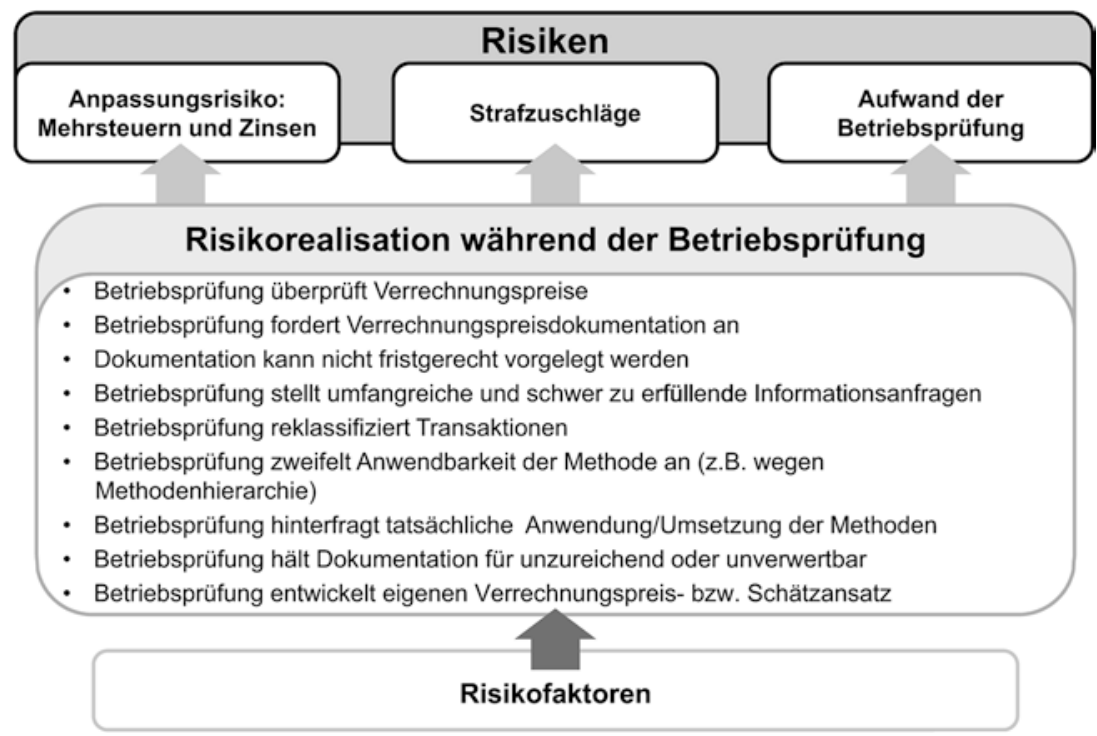

Abb. 6.3 Risikorealisation während der Betriebsprüfung

Im Folgenden soll erörtert werden, wodurch sich die genannten Risiken während einer Betriebsprüfung realisieren. In einem weiteren Schritt sollen die Faktoren identifiziert werden, die die Risikorealisation während der Betriebsprüfung beeinflussen. Schließlich soll bestimmt werden, durch welche Maßnahmen des Betriebsprüfungsmanagements diese Faktoren beeinflusst werden können.

Die Abb. 6.3 gibt einen Überblick über die typischen Ereignisse während der Betriebsprüfung, die die Realisation der genannten Risiken beeinflussen. Diese folgen im Prinzip dem Verlauf einer typischen Betriebsprüfung und deren chronologischer Abfolge:

1. Die Betriebsprüfung entscheidet und kündigt an, dass sie die Verrechnungspreise zu einem Prüfungsfeld der laufenden Betriebsprüfung machen wird.

Diese Entscheidung entzieht sich in der Regel dem direkten Einfluss des Steuerpflichtigen. Die Wahrscheinlichkeit kann aber daran fest gemacht werden, ob der Steuerpflichtige signifikante grenzüberschreitende konzerninterne Transaktionen hat, die der Betriebsprüfung bekannt sind. In der Zukunft ist in diesem Zusammenhang mehr und mehr zu erwarten, dass die Finanzverwaltung die Daten des Steuerpflichtigen vorab screenen wird, um für die Betriebsprüfungen aussichtsreiche Prüfungsfelder zu identifizieren (Stichwort E-Bilanz).

2. Die Betriebsprüfung fordert die Verrechnungspreisdokumentation an und leitet hierfür die relevanten Fristen (60 Tage/30 Tage für außergewöhnliche Geschäftsvorfälle) ein.

Für diesen Punkt gilt ebenfalls, dass er sich in der Regel dem Einfluss des Steuerpflichtigen entzieht und dass die Wahrscheinlichkeit hierfür stark von den Daten hinsichtlich der konzerninternen grenzüberschreitenden Transaktionen abhängig ist. 


\section{Die Dokumentation kann nicht fristgerecht vorgelegt werden.}

Dieser Punkt liegt vollständig in der Hand des Steuerpflichtigen. Er hat die Möglichkeit im Vorfeld der Betriebsprüfung eine Verrechnungspreisdokumentation zu erstellen und sich entsprechend auf die anstehende Betriebsprüfung vorzubereiten.

4. Die Betriebsprüfung stellt umfangreiche und schwer zu erfüllende Informationsanfragen.

In vielen Betriebsprüfungen werden von den Prüfern auch bei Vorlage einer vollständigen, verwertbaren Verrechnungspreisdokumentation zusätzliche Anfragen zum Sachverhalt sowie zur Fremdüblichkeit der Verrechnungspreise gestellt. Dies ist eine normale und rechtlich legitime Vorgehensweise der Betriebsprüfung, die von Amts wegen den Auftrag hat, die Ausführungen des Steuerpflichtigen zu hinterfragen und deren Plausibilität zu überprüfen. Der Steuerpflichtige hat in dieser Phase der Betriebsprüfung die Ermittlungen der Betriebsprüfung durch seine Mitwirkung zu unterstützen. Allerdings ist in manchen Betriebsprüfungen zu beobachten, dass die Betriebsprüfung sehr umfangreiche und nur mit großem Aufwand zu beantwortende Anfragen stellt, die zum Verständnis des Verrechnungspreisansatzes nicht immer erforderlich sind. Dies kann in manchen Fällen für den Steuerpflichtigen zu einem erheblichen Aufwand und zu einer zeitlich ausgedehnten Betriebsprüfung führen. Manchmal werden solche Situationen dadurch befördert, dass der Steuerpflichtige nur stark verzögert oder gar nicht in der Lage ist, Fragen zu beantworten. In anderen Fällen wird die erhöhte Mitwirkungspflicht des Steuerpflichtigen bei Auslandssachverhalten dazu genutzt, um für die Finanzverwaltung ein signifikantes Mehrergebnis zu erzielen. In diesem Zusammenhang stellt sich dann oft die Frage, welche Anfragen noch mit dem Grundsatz der Verhältnismäßigkeit vereinbar sind und wo diese Grenze überschritten wird. Dabei ist es erfahrungsgemäß oft so, dass die Frage hinsichtlich des Umfangs von Anfragen in der Regel schwierig zu beantworten ist. Anders verhält es sich hinsichtlich der Anzahl der zu erstellenden Verrechnungspreisanalysen: Diesbezüglich ist klar, dass der Steuerpflichtige lediglich eine Verrechnungspreisanalyse vorlegen muss, die - sofern sie verwertbar ist -zur Erfüllung der Dokumentationspflichten ausreichend ist. Die Finanzverwaltung kann nicht verlangen, dass der Steuerpflichtige zur Erfüllung der Dokumentationspflichten weitere Analysen anfertigen muss. Dies bedeutet wiederum nicht, dass die Finanzverwaltung nicht eigene Analysen anfertigen und auf deren Basis zu einem abweichenden Ergebnis hinsichtlich der Fremdüblichkeit kommen kann.

5. Die Betriebsprüfung reklassifiziert die konzerninternen Transaktionen des Steuerpflichtigen.

D. h. sie akzeptiert nicht die Transaktionsbedingungen, die der Steuerpflichtige für seine konzerninternen Transaktionen gewählt hat, z. B. nimmt sie statt einer Dienstleistung eine Bereitstellung von Know-how oder anderen immateriellen Wirtschaftsgütern an. Dies geht häufig einher mit der Annahme eines anderen Funktions- und Risikoprofils, als dies vom Steuerpflichtigen angenommen wurde.

6. Die Betriebsprüfung zweifelt die Anwendbarkeit der vom Steuerpflichtigen angewendeten Verrechnungspreismethoden an. 
Hierbei können die Gründe vielfältig sein und von Zweifeln hinsichtlich der tatsächlichen Anwendung der Methode bis hin zur Verneinung der grundsätzlichen Anwendbarkeit der Methoden reichen. Letzteres kann zum Beispiel damit begründet werden, dass die Methodenhierarchie nicht ausreichend berücksichtigt wurde.

7. Die Betriebsprüfung hinterfragt die tatsächliche Anwendung/Umsetzung der Methoden.

Selbst wenn der Steuerpflichtige eine vollständige und verwertbare Dokumentation vorlegt, in der die Anwendbarkeit einer bestimmten Methode für bestimmte Transaktionen analysiert und festgestellt wird, bedeutet dies nicht, dass die Betriebsprüfung regelmäßig die Verrechnungspreise ohne weitere Erörterung akzeptiert.

Ein häufiges Thema bei diesen Erörterungen ist, ob der Steuerpflichtige die Methode tatsächlich wie in der Verrechnungspreisdokumentation beschrieben angewendet hat. In solchen Situationen kann es vorkommen, dass die Betriebsprüfung zahlreiche transaktionsbezogene Einzelnachweise anfragt, um die angesprochene Frage zu analysieren.

\section{Die Betriebsprüfung hält die Dokumentation für unzureichend oder unverwertbar.} Als Einstieg in die Diskussion zwischen dem Steuerpflichtigen und der Betriebsprüfung oder in manchen Fällen auch als Resultat dieser Diskussion kann die Betriebsprüfung zu der Schlussfolgerung kommen, dass die Dokumentation unzureichend und unverwertbar ist. Entsprechend der GAufZV hat die Betriebsprüfung dies unverzüglich festzustellen und dem Steuerpflichtigen mitzuteilen, wenn sie eine Dokumentation für unverwertbar hält. Die Anforderungen an die Unverwertbarkeit sind entsprechend der einschlägigen Kommentierung sehr hoch: Die vorgelegte Dokumentation muss einer Nicht-Dokumentation gleichkommen. In manchen Fällen wird eine teilweise Unverwertbarkeit hinsichtlich der Angemessenheitsdokumentation festgestellt, die daran fest gemacht wird, dass aus Sicht der Betriebsprüfung nicht die richtige bzw. eine nicht akzeptable Methode angewendet wurde. Diese Position ist allerdings in der Regel rechtlich nicht haltbar, wenn der Steuerpflichtige begründen kann, warum er die angewendete Methode für den richtigen Ansatz für die Durchführung bzw. Verprobung des Fremdvergleichs hält.

\section{Die Betriebsprüfung entwickelt einen eigenen Schätzansatz.}

Eine Schätzung ist in der Regel nur zulässig, wenn der Steuerpflichtige seine Dokumentationspflichten bzw. seine Mitwirkungspflichten nicht oder nur unzureichend erfüllt hat. Eine oft zu beobachtende Strategie von Betriebsprüfungen ist es, die Unverwertbarkeit der Verrechnungspreisdokumentation festzustellen, um im weiteren Verlauf die Verrechnungspreise zuungunsten des Steuerpflichtigen schätzen zu können. Der Steuerpflichtige sollte in solchen Situationen - sofern er eine aus seiner Sicht verwertbare Dokumentation vorgelegt hat - darauf verweisen, dass er seine Mitwirkungspflichten erfüllt hat und sich entsprechend gegen eine Schätzung wehren. 


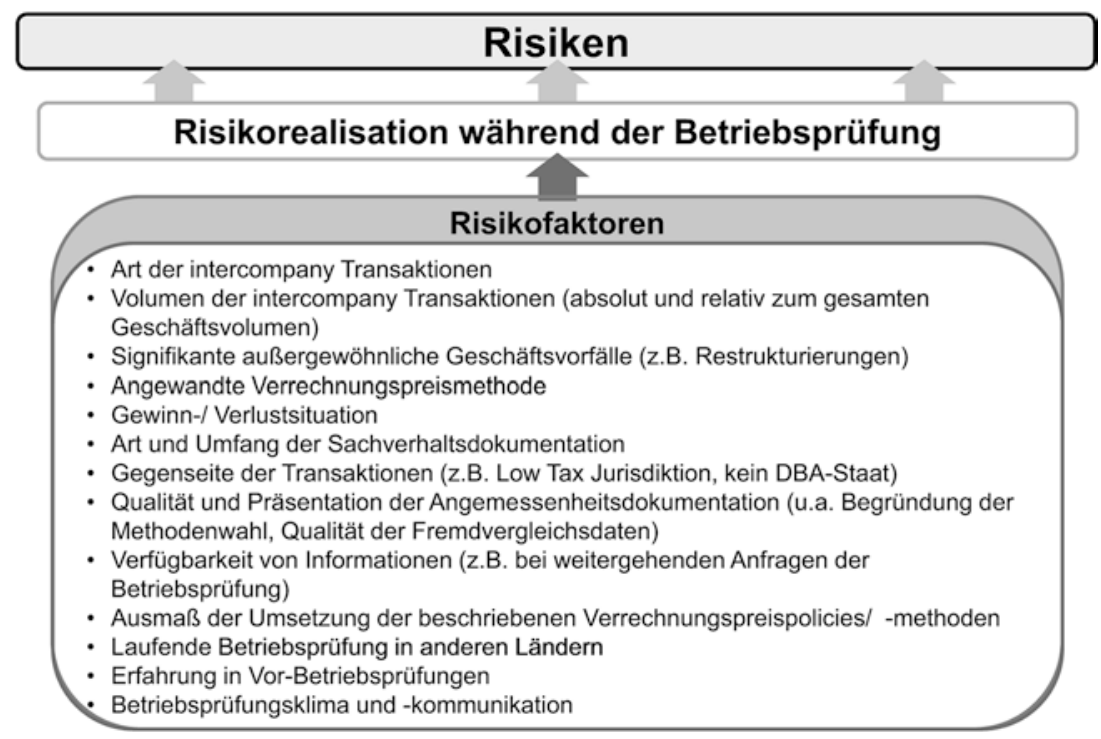

Abb. 6.4 Risikofaktoren

Von welchen Faktoren die Realisation der einzelnen Risikofaktoren und damit der Risiken in einer Betriebsprüfung abhängen, ist in Abb. 6.4 zusammengefasst. Im Folgenden werden die Risikofaktoren jeweils kurz erläutert:

\section{- Art der konzerninternen Transaktionen:}

Bestimmte Arten von Transaktionen stehen stärker im Fokus der Betriebsprüfungen als andere. Während es relativ unstreitig ist, dass für physische Warenlieferungen Preise verrechnet werden müssen, ist dies für Dienstleistungen (bzw. Kostenumlagen), Finanztransaktionen sowie die Bereitstellung bzw. Nutzung immaterieller Wirtschaftsgüter oft nicht unbestritten. Hierbei kommt es in der Regel darauf an, ob eine Outbound- oder eine Inbound-Situation betrachtet wird, d. h. ob über eine Verrechnung von Deutschland ins Ausland oder vom Ausland ins deutsche Inland im Fokus steht.

Insbesondere bei Inbound-Transaktionen wird häufig infrage gestellt, ob überhaupt, dem Grunde nach eine Verrechnung gerechtfertigt ist. Die Betriebsprüfung stellt dann infrage, dass überhaupt Leistungen erbracht wurden, für die fremde Dritte eine Vergütung zahlen würden. Selbst wenn die Verrechnung dem Grunde nach unstrittig ist, kann die Höhe der erhobenen Vergütungen durch die Betriebsprüfung angezweifelt werden. Erfahrungsgemäß kann eine Rechtfertigung für die Verrechnung von immateriellen Wirtschaftsgütern sowie von Dienstleistungen, die physisch nicht wahrnehmbar sind, unter Umständen sehr schwierig sein (vgl. die Ausführungen zur Dokumentation von Dienstleistungen, immateriellen Wirtschaftsgütern sowie Finanztransaktionen in Kap. 3.4 und 5.4). Im Outbound-Fall wird die Frage von der Betriebsprüfung naturgemäß oft umgekehrt gestellt: Wäre eine Verrechnung angesagt gewesen bzw. sind die verrechneten Entgelte hoch genug, um die Leistungen fremdüblich zu kompensieren? 
Ein zunehmend beliebtes Thema ist in diesem Zusammenhang die Verrechnung von Marken-Lizenzen durch die deutsche Konzernzentrale; der Grund ist, dass entsprechende Lizenzgebühren von Betriebsprüfungen in Inbound-Fällen häufig festgestellt werden konnten.

- Volumen der konzerninternen Transaktionen:

Sind die Volumina der konzerninternen Transaktionen im Vergleich zum gesamten Geschäftsvolumen (absolut und relativ) signifikant? Je größer die Transaktionsvolumina sind, umso größer ist das Potenzial für Verrechnungspreisanpassungen durch die Betriebsprüfung, und umso größer ist die Wahrscheinlichkeit, dass die Betriebsprüfung diese Transaktionen genauer prüft.

- Signifikante außergewöhnliche Geschäftsvorfälle (z. B. Restrukturierungen):

Insbesondere außergewöhnliche Geschäftsvorfälle, die einen Einfluss auf zukünftige Geschäftsergebnisse haben, ziehen die Aufmerksamkeit der Betriebsprüfer auf sich. Insofern ist für solche Transaktionen oder Veränderungen die rechtliche Würdigung genau zu prüfen und die erforderliche steuerrechtliche Behandlung zu analysieren. Häufig werden die Anforderungen an eine zeitnahe Dokumentation (spätestens sechs Monate nach Ende des Geschäftsjahres, in dem die Transaktionen bzw. Veränderungen durchgeführt wurden) eingefordert und die Betriebsprüfung erwartet in der Regel, dass eine entsprechende Dokumentation erstellt wurde und vorgelegt werden kann.

- Angewandte Verrechnungspreismethode:

Der Umfang und die Qualität der Analyse der Anwendbarkeit der möglichen Verrechnungspreismethoden in der Verrechnungspreisdokumentation bestimmen, ob die Betriebsprüfung wahrscheinlich die Angemessenheitsanalyse akzeptiert oder infrage stellt. In vielen Fällen muss der Steuerpflichtige mit der Betriebsprüfung umfangreiche Diskussionen hinsichtlich der Anwendbarkeit der gewählten Methode führen. Wenn der Steuerpflichtige gute Argumente, warum seine Methode anwendbar ist, vorbringen kann, sollte er dies in den Diskussionen mit der Betriebsprüfung entsprechend verteidigen und gegebenenfalls weitere unterstützende Argumente für seine Position ins Feld führen. Hinsichtlich dieses Punktes kann eine gewisse Tendenz festgestellt werden, dass die Betriebsprüfung versucht, den methodischen Ansatz des Steuerpflichtigen infrage zu stellen und damit auf die Unverwertbarkeit der Dokumentation, zumindest der Angemessenheitsdokumentation zu schließen. Ziel ist es, in eine Situation zu kommen, in der die Finanzverwaltung die Verrechnungspreise zuungunsten des Steuerpflichtigen schätzen kann. Eine alternative Strategie der Betriebsprüfung zielt darauf ab, festzustellen, dass aufgrund des Fehlens hinreichend vergleichbarer Fremdvergleichsdaten keine der herkömmlichen Verrechnungspreismethoden, weder Standard- noch gewinnorientierte Methoden anwendbar sind. Entsprechend den deutschen Regularien wäre dann lediglich der hypothetische Fremdvergleich anwendbar. Bei der Anwendung des hypothetischen Fremdvergleichs können sich insbesondere wegen der fehlenden Anwendungshinweise in den OECD-RL in der Regel große Spielräume für die Feststellung von Anpassung an den Verrechnungspreisen des Steuerpflichtigen und damit für Steuernachzahlungen ergeben. Für beide Strategien empfiehlt es sich, dass 
der Steuerpflichtige so gut wie möglich versucht, seinen ursprünglichen methodischen Ansatz zu verteidigen.

\section{- Gewinn-/Verlustsituation:}

Verluste im Inland bzw. Gewinne im Ausland sind in der Regel für die Betriebsprüfung ein Indikator, dass die Verrechnungspreise nicht mit dem Fremdvergleichsgrundsatz übereinstimmen. Im Fall von Verlusten wird argumentiert, dass ein unabhängiger Dritter keine längerfristigen Verluste akzeptieren, sondern auf eine Verbesserung der entsprechenden Konditionen (z. B. Senkung der Verrechnungspreise) drängen würde. Hierzu gibt es vom BFH entsprechende Rechtsprechung, die besagt, dass auch ein fremder Dritter für eine gewisse Zeit eine Verlustsituation hinnehmen würde, z. B. in Start-Up Situationen. Hohe Gewinne im Ausland sind insbesondere im Outbound-Fall, wenn also die deutsche Konzernmutter profitable Tochterunternehmen im Ausland hat, ein häufiger Aufgriffspunkt der Betriebsprüfung. In diesem Zusammenhang ist auf das Funktions- und Risikoprofil der betreffenden Unternehmen sowie die beigetragenen wesentlichen (immateriellen) Wirtschaftsgüter abzustellen. Entsprechend ist dann die Fremdüblichkeit der Verrechnungspreise angesichts der übernommenen Funktionen, Risiken und beigetragenen immateriellen Wirtschaftsgüter herauszustellen. Die Profitabilität im Inland oder Ausland wird nicht nur im Zusammenhang mit extrem hohen Gewinnen oder Verlusten thematisiert; oft ist die Profitabilität einer bestimmten Funktion im In- oder Ausland Gegenstand der Diskussion mit der Betriebsprüfung. Dies kann auch dann der Fall sein, wenn - wie häufig von der Betriebsprüfung erwartet - eine Standardmethode, z. B. ein Preisvergleich für die Durchführung des Fremdvergleichs angewendet wurde, die aber aus Sicht der Betriebsprüfung nicht zu einem ausreichenden Gewinn bzw. zu versteuernden Einkommen im Inland führt.

\section{- Qualität und Umfang der Sachverhaltsdokumentation:}

Durch die Dokumentation soll sich die Betriebsprüfung einen Überblick über den für die Verrechnungspreise relevanten Sachverhalt und die Grundlage für die Angemessenheitsanalyse verschaffen. Je besser die Darstellung von den Betriebsprüfern nachvollziehbar ist und die Grundlage für die Angemessenheitsanalyse bildet, umso eher sind die Betriebsprüfer in der Lage der Angemessenheitsanalyse des Steuerpflichtigen zu folgen. In diesem Zusammenhang bedingt ein großer Umfang der Dokumentation nicht notwendigerweise auch eine hohe Qualität, d. h. gute Nachvollziehbarkeit der Dokumentation.

- Gegenseite der Transaktionen (z. B. Low Tax Jurisdiktion, kein DBA-Staat): Ist für die Betriebsprüfung oft ein Indikator, ob die Verrechnungspreise angemessen sind. Transaktionen mit „Niedrigsteuerländern“ stehen eher im Verdacht, dass der Steuerpflichtige möglicherweise über die Gestaltungsspielräume hinaus die Verrechnungspreise zugunsten des Niedrigsteuerlands gewählt hat, um seine Steuerquote zu reduzieren. Länder, mit denen DBAs bestehen (so wie bspw. mit USA und Kanada), die die Möglichkeit eines Schiedsverfahrens beinhalten oder darüber hinausgehende Vereinbarungen (Schiedskonvention innerhalb der EU), sind eher dazu geeignet, ein Verständigungsverfahren bzw. ein Schiedsverfahren im Falle einer Nichteinigung mit der 
Betriebsprüfung als Option in Betracht zu ziehen. Von daher wird die Betriebsprüfung, die in der Regel ein weiteres Verfahren vermeiden und die Betriebsprüfung abschließen möchte, in diesen Fällen eher bemüht sein, einen akzeptablen Kompromiss mit dem Steuerpflichtigen einzugehen, um die Beantragung eines entsprechenden Verfahrens zu vermeiden. Im Gegensatz dazu sind Länder ohne bzw. ohne gut ausverhandeltes DBA eher nicht geeignet, um in der Betriebsprüfung auf ein mögliches internationales Verfahren zu setzen. In diesen Fällen kann die Kompromissfindung mit der Betriebsprüfung unter Umständen schwieriger sein.

- Qualität und Präsentation der Angemessenheitsdokumentation:

Die Begründung der Methodenwahl sowie die Transparenz hinsichtlich der Suche und Analyse der Fremdvergleichsdaten bestimmen, wie gut die Betriebsprüfung die Fremdvergleichsanalyse des Steuerpflichtigen nachvollziehen kann. Je besser die Analyse für einen externen Leser verständlich ist, umso schwieriger ist es für die Betriebsprüfung, mögliche Aufgriffspunkte zu finden. Gleiches gilt für die Qualität der Fremdvergleichsdaten, d. h., wie hoch der Grad der Vergleichbarkeit zu den konzerninternen Transaktionen bzw. Transaktionsströmen ist. Allerdings gibt es in der Regel keine hundertprozentige Vergleichbarkeit. Dabei kommt es wesentlich auf die gewählte Verrechnungspreismethode an, wie hoch der Grad der Vergleichbarkeit sein sollte - bei Standardmethoden sind die Anforderungen höher als bei gewinnorientierten Methoden (vgl. Kap. 5.3.1).

- Verfügbarkeit von Informationen (z. B. bei weitergehenden Anfragen der Betriebsprüfung):

Die Betriebsprüfung wird sich regelmäßig nicht auf die Durchsicht der Verrechnungspreisdokumentation beschränken. Vielmehr wird sie weitere Fragen zum Sachverhalt und zur Angemessenheitsanalyse haben und gegebenenfalls weitere Dokumente, Finanzdaten sowie Daten zu den Transaktionen anfragen, um die Plausibilität der Ausführungen in der Verrechnungspreisdokumentation zu hinterfragen. Solche Anfragen sind für den Prüfungsauftrag der Betriebsprüfung erforderlich und sollten daher vom Steuerpflichtigen auch so gut wie möglich bedient werden. Der Steuerpflichtige sollte allein schon deshalb versuchen, die Anfragen so gut wie möglich zu beantworten, weil er insbesondere im Zusammenhang mit Auslandssachverhalten zur Mitwirkung in der Betriebsprüfung verpflichtet ist und eine mangelnde Mitwirkung von der Betriebsprüfung unter bestimmten Bedingungen zum Anlass genommen werden kann, die Verrechnungspreise zuungunsten des Steuerpflichtigen zu schätzen (vgl. die Ausführungen zur Verhältnismäßigkeit von Informationsanfragen und die Möglichkeiten zur Schätzung durch die Betriebsprüfung oben).

- Ausmaß der Umsetzung der beschriebenen Verrechnungspreispolicy/-methoden: Der in der Verrechnungspreisanalyse beschriebene Verrechnungspreisansatz sollte mit den tatsächlich umgesetzten Verrechnungspreisen bzw. deren Ergebnissen übereinstimmen. Beispielsweise ist es sicherlich nicht günstig für die Argumentation des Steuerpflichtigen in der Betriebsprüfung, wenn in der Verrechnungspreisanalyse einerseits festgestellt wird, dass für eine bestimmte Funktion eine Vergütung bzw. Marge 
innerhalb einer bestimmten Bandbreite angemessen ist, wenn die tatsächliche Marge, die für diese Funktion erzielt wurde, außerhalb der genannten Bandbreite liegt.

\section{- Laufende Betriebsprüfung in anderen Ländern:}

Die Betriebsprüfer fragen regelmäßig nach Ergebnissen und Anpassungen in den Betriebsprüfungen in den Ländern, mit dem der Steuerpflichtige die konzerninternen Transaktionen durchführt. Diese können eine Indikation geben, ob die Verrechnungspreise in den betreffenden Transaktionen von den jeweiligen ausländischen Finanzverwaltungen als angemessen, d. h. fremdüblich angesehen wurden.

\section{- Erfahrung in Vorbetriebsprüfungen:}

Diese geben einerseits einen Hinweis darauf, wie die jeweiligen Betriebsprüfer mit den Verrechnungspreisthemen des Unternehmens in der Vergangenheit umgegangen sind. Darüber hinaus gibt es häufig Sachverhalte, die sich über mehrere Betriebsprüfungszeiträume fortsetzen und die daher von der Betriebsprüfung im Folgezeitraum wahrscheinlich in der gleichen Weise behandelt werden, wie in zuvor geprüften Jahren. Insofern können Feststellungen in der Vorbetriebsprüfung einen Hinweis darauf geben, wie hoch das Risiko in der folgenden Betriebsprüfung sein kann. Da die Betriebsprüfer wechseln können und weil diese rechtlich nicht durch die Vorbetriebsprüfung gebunden sind, bedeutet dies nicht, dass die Nichtbeanstandung eines Verrechnungspreisansatzes zu Rechtssicherheit für die nachfolgende Betriebsprüfung führt.

\section{- Betriebsprüfungsklima und -kommunikation:}

Dies ist ein ganz wesentlicher Faktor, den der Steuerpflichtige stark beeinflussen kann und der einen großen Einfluss auf das Ergebnis einer Betriebsprüfung hat. Dabei steht im Vordergrund, dass der Steuerpflichtige für eine angenehme, professionelle sowie sachlich bestimmte Atmosphäre sorgen sollte. Der Steuerpflichtige sollte regelmäßig mit den Betriebsprüfern Besprechungen durchführen, in denen der Betriebsprüfungsablauf, die offenen Fragen sowie die strittigen Punkte besprochen werden. Zur Dokumentation des Betriebsprüfungsprozesses empfiehlt es sich, die Anfragen der Betriebsprüfung sowie die Antworten vonseiten des Steuerpflichtigen schriftlich festzuhalten, auch wenn der eigentliche Austausch der Dokumente und Antworten dann in mündlichen Besprechungen stattfinden sollte, um persönlich über die verschiedenen Positionen ein gemeinsames Verständnis herzustellen. Dabei hat der Steuerpflichtige im Prinzip einen Anspruch auf schriftlich formulierte Prüfungsanfragen vonseiten der Betriebsprüfung. Gleichzeitig ist es von großer Bedeutung, dass der Verantwortliche des Steuerpflichtigen für die Betriebsprüfungen einen Überblick über die Ansprechpartner hat, mit denen die Betriebsprüfung spricht, und über die Informationen, die an die Betriebsprüfung weitergegeben werden. Dies gilt im Allgemeinen für das Betriebsprüfungsmanagement für alle steuerlichen Themen.

Für den Bereich der Verrechnungspreise ist von besonderer Bedeutung, dass sich mittlerweile sowohl aufseiten der Finanzverwaltung als auch aufseiten der Steuerpflichtigen mehr und mehr Experten gegenüberstehen. So ist es mittlerweile gängige Praxis, dass das Spezialthema Verrechnungspreise in Besprechungen zwischen den Experten für dieses Thema separat diskutiert wird. Dies macht in der Regel Sinn, um ein 


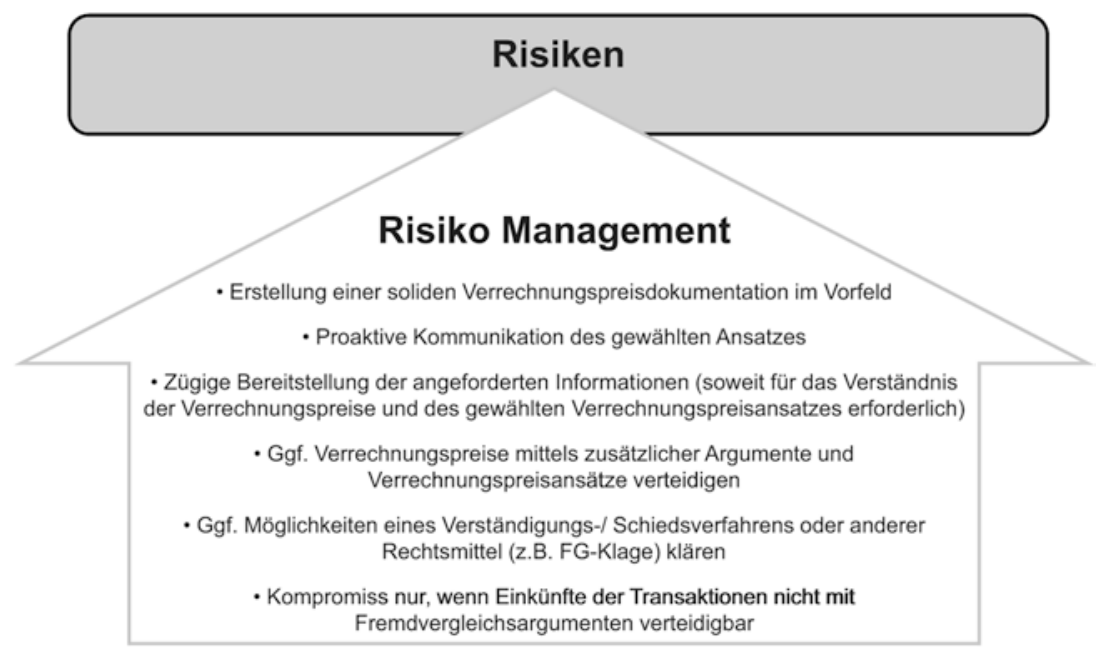

Abb. 6.5 Risikomanagement

gemeinsames Verständnis über den Sachverhalt und dessen Würdigung aus Verrechnungspreissicht zu erarbeiten, was eine Grundvoraussetzung für eine spätere Einigung zwischen den Parteien darstellt.

Als Konsequenz aus den Erfahrungen mit den Risikofaktoren resultieren folgende Empfehlungen für eine Best-Practice für ein effizientes Betriebsprüfungsmanagement. Diese Empfehlungen sind geeignet, die genannten Risiken einer Betriebsprüfung der Verrechnungspreise wesentlich zu minimieren (Abb. 6.5).

Die Empfehlungen werden im Folgenden erläutert.

\section{- Erstellung einer soliden Verrechnungspreisdokumentation im Vorfeld:}

Es empfiehlt sich, die Betriebsprüfung frühzeitig vorzubereiten und die Dokumentation und Verteidigung der Verrechnungspreise frühzeitig anzugehen. Nicht empfehlenswert ist es, erst zu warten, bis die Dokumentation von der Betriebsprüfung angefragt wird. Zwar ist es innerhalb der 60-Tage-Frist zumeist auf irgendeine Weise möglich, eine Dokumentation zu erstellen, die den Mindestanforderungen entspricht. Dies gilt aber auch nur unter der Bedingung, dass die Transaktionen nicht zu komplex und alle Informationen sofort verfügbar sind. Erfahrungsgemäß ist aber die Informationsbeschaffung umso schwieriger, je weiter die Sachverhalte zurück liegen. Zudem braucht eine fundierte Angemessenheitsanalyse auch eine gewisse Zeit und kann nicht immer mit Studien aus dem Fundus eines Experten erledigt werden, zumal das Ergebnis möglicherweise nicht zufriedenstellend ist und zu Diskussionen mit der Betriebsprüfung führen kann. Die meisten Schwierigkeiten hinsichtlich der Unverwertbarkeit der Dokumentation resultieren in Situationen, in denen die Dokumentation kurzfristig während der Anforderungsfrist erstellt wird. Dies wird sich mit den neuen 
OECD-Dokumentationsanforderungen im Rahmen von BEPS und den entsprechenden kurzen Vorlagefristen verschärfen. ${ }^{21}$

Die Steuerpflichtigen unterliegen Ressourcenrestriktionen und die Erstellung der Verrechnungspreisdokumentation muss effizient und daher so kostensparend wie möglich erstellt werden. Dabei ist eine High-end-Version einer Verrechnungspreisdokumentation oft auch nicht erforderlich. Vielmehr sind eine gut recherchierte Sachverhaltsdokumentation und eine fundierte Fremdvergleichsanalyse die beste Vorbereitung für die Diskussion mit der Betriebsprüfung. Sie stellt daher in den meisten Fällen eine lohnende Investition dar, da sie eine Menge Aufwand und schwierige Diskussionen mit der Betriebsprüfung vermeiden kann. In Zukunft ist zudem darauf zu achten, dass die neuen OECD-Anforderungen, die sich aus den BEPS Action Items 8-10 ergeben, eine umfassendere und genauere Dokumentation hinsichtlich der Wertschöpfungskette und der ökonomischen Substanz (z. B. wer kontrolliert bestimmte Prozesse) erfordern (vgl. hierzu Kap. 3, 4 und 5). ${ }^{22}$

Keinesfalls auf eine mögliche Erstellung während der Vorlagefrist verlassen sollte sich der Steuerpflichtige bei den außergewöhnlichen Geschäftsvorfällen, die zeitnah zu dokumentieren sind und für die die Vorlagefrist lediglich 30 Tage beträgt. Eine solide Dokumentation kann innerhalb der Frist von 30 Tagen noch weniger gut erstellt werden als in 60 Tagen. Hinzu kommt, dass sich rechtlich negative Konsequenzen ergeben können, weil die Betriebsprüfer wegen der nicht zeitnah erstellten Dokumentation noch eher dazu tendieren, eine Schätzung vornehmen zu wollen, auch wenn dies rechtlich meist nicht gerechtfertigt ist.

- Proaktive Kommunikation des gewählten Ansatzes:

Bereits zu Beginn der Betriebsprüfung sollte der Steuerpflichtige, falls die Betriebsprüfung die Dokumentation angefordert hat, die Sachverhaltsdokumentation und die Angemessenheitsanalysen der Betriebsprüfung präsentieren und erläutern, damit diese mit den gewählten Ansätzen des Steuerpflichtigen vertraut gemacht werden. Dies kann am besten in einer Besprechung mit der Betriebsprüfung bei Übergabe der Dokumentation erfolgen. Erste Fragen der Betriebsprüfung zum Sachverhalt und zur Angemessenheitsanalyse können bereits in dieser Besprechung adressiert werden. Diese Besprechung ist in der Regel essentiell, um die Grundlage für eine konstruktive Diskussion über die vom Steuerpflichtigen gewählten Ansätze zu schaffen und ein gutes Betriebsprüfungsklima herzustellen.

- Zügige Bereitstellung der angeforderten Informationen soweit für das Verständnis der Verrechnungspreise und des gewählten Verrechnungspreisansatzes erforderlich:

Weitere Fragen der Betriebsprüfung sollten soweit möglich zügig, vollständig und konstruktiv beantwortet werden. Dies ist in der Regel für die Herstellung und den Erhalt eines guten Betriebsprüfungsklimas von großer Bedeutung. Die Fragen, die von

${ }^{21}$ OECD (2015b), Transfer Pricing Documentation and Country-by-Country Reporting.

22 OECD (2015a), Aligning Transfer Pricing Outcomes with Value Creation. 
der Betriebsprüfung schriftlich gestellt werden, sollten vom Steuerpflichtigen auch schriftlich beantwortet werden. Die Übergabe der schriftlichen Antworten sollte am besten in Besprechungen mit der Betriebsprüfung erfolgen, damit ggfs. ein Verständnis des Hintergrunds für die Fragestellung der Betriebsprüfung entwickelt werden kann. Der Steuerpflichtige kann in diesen Besprechungen gegebenenfalls weitere Erläuterungen geben und weitergehende Fragen der Betriebsprüfung direkt adressieren. Dabei kann er in diesen Besprechungen seine Position auf konstruktive Weise deutlich machen und mit der Betriebsprüfung diskutieren, bevor Feststellungen vonseiten der Betriebsprüfungen getroffen werden. Sollte sich abzeichnen, dass Anfragen der Betriebsprüfung unverhältnismäßig umfangreich und obendrein für das Verständnis des Verrechnungspreisansatzes nicht erforderlich sind, sind die Besprechungen eine gute Gelegenheit, um der Betriebsprüfung diesen Standpunkt zu vermitteln.

- Verrechnungspreise mittels zusätzlicher Argumente und Verrechnungspreisansätze verteidigen:

Zeichnet sich ab, dass die Betriebsprüfung den Verrechnungspreisansatz des Steuerpflichtigen nicht akzeptieren und Anpassungen an den Verrechnungspreisen bzw. am zu versteuernden Ergebnis vornehmen will, sollte der Steuerpflichtige -sofern er einen soliden Ansatz vorgelegt hat- seinen Ansatz auf Basis der zugrunde liegenden Analyse verteidigen. Wo Zweifel an der Sachverhaltsanalyse oder dem Verrechnungspreisansatz vonseiten der Betriebsprüfung geäußert werden, sollten gegebenenfalls weitere Ermittlungen und Analysen durchgeführt werden, um die Position des Steuerpflichtigen weiter zu untermauern. Dies mag auch angesagt sein, wenn die Betriebsprüfung eigene Verrechnungspreisansätze (z. B. Datenbankanalysen oder die Anwendung eines alternativen methodischen Ansatzes) durchführt. In der Regel empfiehlt es sich, die zusätzlichen Argumente und Analysen in schriftlichen Stellungnahmen an die Betriebsprüfung zu adressieren. Diese sollten dann gegebenenfalls wieder in Besprechungen mit den Betriebsprüfern im direkten Gespräch erläutert und mit der Betriebsprüfung diskutiert werden.

- Möglichkeiten eines Verständigungs-/Schiedsverfahrens oder anderer Rechtsmittel (z. B. Finanzgerichtsklage) klären:

Wird im Verlauf des Austauschs mit der Betriebsprüfung deutlich, dass diese eine signifikante Anpassung an den Verrechnungspreisen bzw. dem zu versteuernden Einkommen durchzuführen beabsichtigt, sollte der Steuerpflichtige mögliche Optionen für die Beseitigung der Doppelbesteuerung in einem Verständigungsverfahren/Schiedsverfahren oder einer Klage gegen eine entsprechende Festsetzung in einem Finanzgerichtsverfahren ausloten. Dies sollte vor Ende der Schlussbesprechung erfolgen, da die Optionen bei Nichteinigung entscheidend für die Verhandlungsstrategie des Steuerpflichtigen sind. Die Optionen bei Nichteinigung (Outside-Options) sind ein wichtiger Faktor zur Beantwortung der Frage, bis zu welcher Höhe der Steuerpflichtige einen Kompromiss akzeptieren sollte. Erfahrungsgemäß bietet sich im Verrechnungspreisbereich eher die Beantragung eines Verständigungsverfahrens an, da hierdurch die Doppelbesteuerung vermieden werden kann, unter der Voraussetzung, dass eine 
korrespondierende Anpassung im Land der Gegenseite der Transaktion tatsächlich zu einer Steuerentlastung für die Unternehmensgruppe führt. Dies ist bei einem Finanzgerichtsverfahren keinesfalls sicher. Ein Finanzgerichtsverfahren bietet sich in der Regel dann an, wenn es bei den von der Betriebsprüfung aufgeworfenen Fragen primär um nationale Regularien geht, z. B. die Verwertbarkeit der Dokumentation und mögliche Strafzuschläge und/oder wenn eine korrespondierende Anpassung nicht zu einer steuerlichen Entlastung auf der Gegenseite der Transaktion führt. (vgl. hierzu auch Kap. 2.4.5 Methoden zur Vermeidung der Doppelbesteuerung)

- Kompromiss nur, wenn die Verrechnungspreise offensichtlich nicht mit Fremdvergleichsargumenten verteidigbar sind:

Erst wenn alle Argumente zu Genüge ausgetauscht wurden und sich eine Einigung bzw. Nichteinigung abzeichnet, sollten die Parteien in die finale Phase der Betriebsprüfung eintreten und die Schlussbesprechung durchführen. In dieser werden die jeweiligen Positionen noch einmal zusammengefasst und gegenübergestellt. Dabei werden die Möglichkeiten für eine Einigung ausgelotet. Zum Ende der Betriebsprüfung wird in der Regel über eine finale Einigung verhandelt. Dabei sollte sich der Steuerpflichtige nur auf einen Kompromiss einlassen, sofern dieser angesichts der Optionen bei einer Nichteinigung (s. vorheriger Abschnitt) der Höhe nach akzeptabel ist. Streng genommen sollte sich der Steuerpflichtige nur auf einen Kompromiss mit der Betriebsprüfung einlassen, wenn die Verrechnungspreisansätze in den betreffenden Transaktionen nicht mittels Fremdvergleichsargumenten untermauert werden kann. In der Praxis einigt man sich oft trotz eines verteidigungsfähigen Ansatzes des Steuerpflichtigen auf eine Anpassung, sofern daraus resultierende Kosten (Steuern, Zinsen) die antizipierten Kosten eines Verständigungs- oder Finanzgerichtsverfahrens übersteigen. Hierbei sind ggfs. auch Folgeeffekte im Zeitraum nach der Betriebsprüfung in Betracht zu ziehen. Solche Effekte können dadurch entstehen, dass die Änderung des Ansatzes durch die Betriebsprüfung auf die nachfolgenden Zeiträume übertragen wird.

Die Empfehlungen verstehen sich als verallgemeinerte Quintessenz aus zahlreichen Betriebsprüfungen. Es ist jeweils im Einzelfall die spezifische Situation zu beurteilen und auf dieser Basis über die beste Betriebsprüfungsstrategie zu entscheiden. Hierbei spielen die spezifischen Umstände des Unternehmens (die Risikofaktoren) aber auch die jeweilige Konstellation aufseiten der Betriebsprüfung eine entscheidende Rolle.

\section{Literatur}

BFH, Urteil vom 17.10.2001 - Az. I R 103-00. Konzernverrechnungspreise als vGA einer Vertriebsgesellschaft. BStB1 II 2004, S. 171

BMF, Schreiben vom 22.10.2015. Ergebnisse der steuerlichen Betriebsprüfung 2014

BMF, Schreiben vom 30.12.1999. Verwaltungsgrundsätze Umlageverträge. BStB1 I 1999, S. 1122 (VWG-Ulageverträge)

BMF, Schreiben vom 12.4.2005. Grundsätze für die Prüfung der Einkunftsabgrenzung zwischen nahestehenden Personen mit grenzüberschreitenden Geschäftsbeziehungen in Bezug auf 
Ermittlungs- und Mitwirkungspflichten, Berichtigungen sowie auf Verständigungs- und EU Schiedsverfahren (VWG-Verfahren). IV B 4- S 1341-1/05. BStB1 2005 I, S. 570

Europäische Kommission, EU Joint Transfer Pricing Forum. 2004. Is Europe One Market? A Transfer Pricing Economic Analysis of Pan-European Comparables Sets. http://ec.europa.eu/taxation customs/resources/documents/forum7/europe_one_market_white_paper_feb18.pdf.

EU Joint Transfer Pricing Forum, Discussion Paper on Scoping the Work on Comparables in the EU, February 2016, („EU JTPF 2016 Working Paper on Comparables“) http://ec.europa. eu/taxation_customs/resources/documents/taxation/company_tax/transfer_pricing/forum/ jtpf/2016/20160218_comparables.pdf

Kuckhoff H., Schreiber R. 1997, Verrechnungspreise in der Betriebsprüfung, Beck, München, VII

OECD 22. Juli, 2010. Transfer Pricing Guidelines for Multinational Enterprises and Tax Administrations. OECD Publishing (OECD-RL)

OECD (2015a), Aligning Transfer Pricing Outcomes with Value Creation, Actions 8-10 - 2015 Final Reports, OECD/G20 Base Erosion and Profit Shifting Project, OECD Publishing, Paris („OECD (2015), Aligning Transfer Pricing Outcomes with Value Creation“) http://dx.doi.org/10.1787/ 9789264241244-en

OECD (2015b), Transfer Pricing Documentation and Country-by-Country Reporting, Action 13 2015 Final Reports, OECD/G20 Base Erosion and Profit Shifting Project, OECD Publishing, Paris („OECD (2015), Transfer Pricing Documentation and Country-by-Country Reporting“) http://dx.doi.org/10.1787/9789264241480-en

PricewaterhouseCoopers AG Wirtschaftsprüfungsgesellschaft 2015, Betriebsprüfung 2015 („PwC, Betriebsprüfung 2015“)

Schreiber, R. Verwaltungsgrundsätze Verfahren. In Handbuch Internationale Verrechnungspreise, hrsg. Kroppen, H.-K. Dr. Otto Schmidt Verlag, Köln

Schoppe, C. \& Stumpf, B. 2014, Was sind „im Wesentlichen unverwertbare“ Aufzeichnungen, BB, S. 1116

Wassermeyer, F. in Außensteuerrecht Kommentar $§ 1$ AStG, hrsg. Flick, Wassermeyer, F., Baumhoff, H., Schönfeld, J. Dr. Otto Schmidt Verlag Köln

Dr. Thomas Bittner ist Senior Manager im Bereich Verrechnungspreise bei PwC am Standort Hamburg. Er hat an der Universität Münster Volkswirtschaftslehre studiert und dort promoviert. $\mathrm{Zu}$ seinen Schwerpunkten zählen neben der Planung, Dokumentation und Verteidigung von Verrechnungspreisen die Analyse von immateriellen Wirtschaftsgütern im Zusammenhang mit Verrechnungspreisen.

Dr. Roman Dawid ist seit Ende 2013 als Partner im Bereich Verrechnungspreise bei PwC am Standort Frankfurt am Main tätig. Er hat seine Laufbahn als Berater im Bereich der Verrechnungspreise 2001 bei Deloitte begonnen. Seitdem hat er eine breite Expertise in Verrechnugspreisprojekten für zahlreiche in- und ausländische Unternehmen vom Mittelständler bis hin zu großen börsengelisteten Unternehmensgruppen in verschiedenen Industrien (u.a. Industriegüter, Konsumgüter, Technology, sowie Automotive) erworben. Von 2005 bis 2013 hat er das Team der Service Line Verrechnungspreise von Deloitte am Standort Frankfurt am Main aufgebaut und geleitet. Dort wurde er 2007 zum Director und 2009 zum Partner bestellt. Roman Dawid berät internationale Unternehmensgruppen bei der Strukturierung ihrer Verrechnungspreissysteme, sowie deren Dokumentation und Verteidigung in Betriebsprüfungen. In diesem Bereich ist er insbesondere spezialisiert auf die ökonomische Analyse von Wertschöpfungsketten sowie die Vergütung immaterieller Wirtschaftsgüter. Darüber hinaus hat er zahlreiche Verständigungsverfahren und Advanced Pricing Agreements begleitet. Roman Dawid hat eine vielfältige Liste an Publikationen und ist u.a. Autor des „Handbuchs Internationale Verrechnungspreise“, das von Prof. Heinz-Klaus Kroppen herausgegeben wird. Er wird seit 
mehreren Jahren im Euromoney Guide to the World's Leading Transfer Pricing Advisers als einer der weltweit führenden Verrechnungspreisberater geführt. Er ist Diplom-Volkswirt und hat an den Universitäten Marburg und Konstanz studiert. Anschließend promovierte er an der Ruhr-Universität Bochum am Lehrstuhl für öffentliche Finanzen und Steuern, wo er vor seiner Beratertätigkeit mehrere Jahre als wissenschaftlicher Mitarbeiter tätig war. Seit dem Wintersemester 2014 hat er einen Lehrauftrag an der Georg-August-Universität Göttingen im Master of Taxation Programm übernommen.

Susann Metzner ist bei Flick Gocke Schaumburg am Standort München tätig. Zuvor arbeitete sie als Managerin bei Deloitte am Standort Frankfurt am Main. Frau Metzner ist Steuerberaterin und Dipl.-Volkswirtin (Int.) und hat an der Universität Erlangen-Nürnberg und der PUC Rio de Janeiro, Brasilien, Internationale Volkswirtschaftslehre studiert. Sie berät multinationale Unternehmen bei der Errichtung, Implementierung und Dokumentation von globalen Verrechnungspreissystemen und unterstützt internationale Konzerne bei der Verteidigung der Verrechnungspreissysteme.

Open Access Dieses Kapitel wird unter der Creative Commons Namensnennung-Nicht kommerziell 2.5 International Lizenz (http://creativecommons.org/licenses/by-nc/2.5/deed.de) veröffentlicht, welche für nicht kommerzielle Zwecke die Nutzung, Verbreitung und Wiedergabe in jeglichem Medium und Format erlaubt, sofern Sie den/die ursprünglichen Autor(en), den Titel des Werks und die Quelle ordnungsgemäß nennen, einen Link zur Creative Commons Lizenz beifügen und im Falle einer Abwandlung durch einen entsprechenden Hinweis deutlich erkennbar machen, dass Änderungen vorgenommen wurden.

Die in diesem Kapitel enthaltenen Bilder und sonstiges Drittmaterial unterliegen ebenfalls der genannten Creative Commons Lizenz, sofern sich aus der Abbildungslegende nichts anderes ergibt. Sofern das betreffende Material nicht unter der genannten Creative Commons Lizenz steht und die betreffende Handlung nicht nach gesetzlichen Vorschriften erlaubt ist, ist auch für die oben aufgeführten nichtkommerziellen Weiterverwendungen des Materials die Einwilligung des jeweiligen Rechteinhabers einzuholen. 